\title{
A viral perspective on worldwide non- pharmaceutical interventions against COVID-19
}

Jean-Philippe Rasigade ( $\sim$ jean-philippe.rasigade@chu-lyon.fr )

University of Lyon https://orcid.org/0000-0002-8264-0452

\section{Anaïs Barray}

University of Lyon

\section{Julie Teresa Shapiro}

Claude Bernard University Lyon 1 https://orcid.org/0000-0002-4539-650X

\section{Charlène Coquisart}

Muséum national d'Histoire naturelle

\section{Yoann Vigouroux}

Muséum national d'Histoire naturelle

\section{Antonin Bal}

Hospices Civils de Lyon - Centre International de Recherche en Infectiologie

\section{Grégory Destras}

University of Lyon

\section{Philippe Vanhems}

Hospices Civils de Lyon

\section{Bruno Lina}

Université Lyon I

\section{Laurence Josset}

University of Lyon

\section{Thierry Wirth}

Museum National d'Histoire Naturelle https://orcid.org/0000-0002-7371-0172

\section{Article}

Keywords: COVID-19, non-pharmaceutical interventions, SARS-CoV-2

Posted Date: November 5th, 2020

DOl: https://doi.org/10.21203/rs.3.rs-101080/v1

License: (c) (i) This work is licensed under a Creative Commons Attribution 4.0 International License. Read Full License 


against COVID-19

3 Jean-Philippe Rasigade ${ }^{1,2^{*}}$, Anaïs Barray ${ }^{1}$, Julie Teresa Shapiro ${ }^{1}$, Charlène Coquisart ${ }^{3,4}$, Yoann Vigouroux $^{3,4}$, Antonin Bal ${ }^{1,2,5}$, Grégory Destras ${ }^{1,2,5}$, Philippe Vanhems ${ }^{1,6}$, Bruno Lina ${ }^{1,2,5}$, Laurence Josset ${ }^{1,2,5}$, Thierry Wirth ${ }^{3,4^{*}}$.

${ }^{1} \mathrm{CIRI}$, Centre International de Recherche en Infectiologie, Université de Lyon, Inserm U1111, $8 \quad$ Université Claude Bernard Lyon 1, CNRS UMR5308, Ecole Normale Supérieure de Lyon, Lyon 9 69007, France.

$10 \quad 2$ Institut des Agents Infectieux, Hospices Civils de Lyon, Lyon 69004, France.

$11{ }^{3}$ Institut de Systématique, Evolution, Biodiversité (ISYEB), Muséum national d'Histoire naturelle, 12 CNRS, Sorbonne Université, Université des Antilles, EPHE, Paris 75005, France.

$13 \quad{ }^{4}$ PSL University, EPHE, Paris 75014, France.

$14 \quad{ }^{5}$ Centre National de Référence des Virus Respiratoires, Hospices Civils de Lyon, Lyon 69004, 15 France. (HCL), Lyon 69008, France.

${ }^{*}$ Corresponding author: jean-philippe.rasigade@univ-lyon1.fr; alternate corresponding author: wirth@mnhn.fr 
Abstract:

23 Quantifying the effectiveness of large-scale non-pharmaceutical interventions (NPIs) 24 against COVID-19 is critical to adapting responses against future waves of the 25 pandemic. Most studies of NPIs thus far have relied on epidemiological data. Here, we 26 report the impact of NPIs on the evolution of SARS-CoV-2, taking the perspective of the 27 virus. We examined how variations through time and space of SARS-CoV-2 genomic 28 divergence rates, which reflect variations of the epidemic reproduction number Rt, can 29 be explained by NPIs and combinations thereof. Based on the analysis of 5,198 SARS30 CoV-2 genomes from 57 countries along with a detailed chronology of 9 non31 pharmaceutical interventions during the early epidemic phase up to May 2020, we find 32 that home containment (35\% Rt reduction) and education lockdown $(26 \%)$ had the 33 strongest predicted effectiveness. To estimate the cumulative effect of NPIs, we 34 modelled the probability of reducing Rt below 1 , which is required to stop the epidemic, 35 for various intervention combinations and initial Rt values. In these models, no 36 intervention implemented alone was sufficient to stop the epidemic for Rt's above 2 and 37 all interventions combined were required for Rt's above 3. Our approach can help 38 inform decisions on the minimal set of NPIs required to control the epidemic depending 39 on the current Rt value. 
Coronavirus disease 2019 (COVID-19), caused by the severe acute respiratory syndrome coronavirus 2 (SARS-CoV-2), emerged in China in late 20191-3. Facing or anticipating the pandemic, the governments of most countries implemented a wide range of large-scale non-pharmaceutical interventions, such as lockdown measures, in order to reduce COVID-19 transmission ${ }^{4-6}$. Concerns have been raised regarding the impact of such interventions on the economy, education, and, indirectly, the healthcare system ${ }^{7}$.

Understanding the effectiveness of each non-pharmaceutical intervention against COVID-19 is critical to implementing appropriate responses against current or future waves of the pandemic. Comparative studies of interventions typically rely on epidemiological data to estimate variations of the epidemic reproduction number, which are then correlated with the implementation or relaxation of interventions ${ }^{5,6,8}$. These studies yielded conflicting conclusions. Depending on data sources and epidemiological model design assumptions, some studies identified lockdown (stay at home order) as the most effective intervention ${ }^{5,9}$ while others found little additional impact, if any, compared to other interventions ${ }^{4,6,10}$. Epidemiological studies of interventions against an epidemic face several challenges. Models informed by counts of confirmed cases or deaths ignore the relationships and transmission patterns between cases. Counts themselves can vary in accuracy and timeliness depending on countries' health facilities, surveillance systems, and the changing definitions of cases. Even when an intervention immediately reduces the transmission rate, a detectable reduction of disease incidence can be much delayed ${ }^{6}$, especially when testing and diagnoses are restricted to specific patient populations. This delay from intervention to incidence reduction, combined with the variety and simultaneous implementation of interventions ${ }^{4,5}$, complicates the identification of their individual effects.

Unlike epidemiological case counts, viral genomes bear phylogenetic information relevant to disease transmission. Extracting this information is the goal of phylodynamics, which relies on evolutionary theory and bioinformatics to model the dynamics of an epidemic ${ }^{11}$. The dates of viral transmission events can also be inferred from genome sequences to alleviate, at least in part, the problems of delayed detection 
70 of an intervention's effect. Here, we conducted a phylodynamic analysis of 5,198 SARS-

71 CoV-2 genomes from 57 countries to estimate the independent effects of 9 large-scale

72 non-pharmaceutical interventions on the transmission rate of COVID-19 during the early

73 dissemination phase of the pandemic. We adapted an established phylogenetic

74 method $^{12,13}$ to model variations of the divergence rate of SARS-CoV-2 in response to

75 interventions and combinations thereof. Building on known relationships between the

76 viral divergence rate and the effective reproduction number $R_{t}{ }^{14}$, we quantified the

77 reduction of $R_{t}$ independently attributable to each intervention, exploiting

78 heterogeneities in their nature and timing across countries in multivariable models. In

79 turn, these results enabled estimating the probability of stopping the epidemic $\left(R_{t}<1\right)$

80 when implementing selected combinations of interventions. 


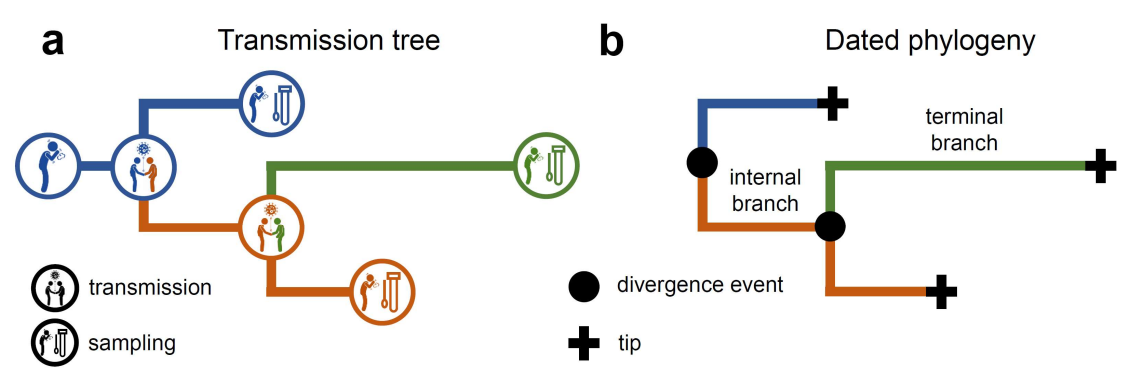

Fig. 1. Conceptual overview of phylodynamic survival analysis. Under the assumption that each viral lineage in a phylogeny belongs to an infected patient, the dates of viral transmission and sampling events in a transmission tree (a) coincide with the dates of divergence events (nodes) and tips, respectively, of the dated phylogeny reconstructed from the viral genomes (b). Treating viral transmission as the event of interest for survival modelling, internal branches connecting two divergence events are interpreted as time-to-event intervals while terminal branches, that do not end with a transmission event, are interpreted as censored intervals (c). Translating the dated through time as a Kaplan-Meier curve (d) and modelling the transmission rate using Cox proportional hazards regression. 


\section{Survival modelling of viral transmission}

101 The dissemination and detection of a virus in a population can be described as a 102 transmission tree (Fig. 1a) whose shape reflects that of the dated phylogeny of the 103 sampled pathogens (Fig. 1b). In a phylodynamic context, it is assumed that each 104 lineage, represented by a branch in the phylogenetic tree, belongs to a single patient 105 and that lineage divergence events, represented by tree nodes, coincide with 106 transmission events ${ }^{11}$. Thus, branches in a dated phylogeny represent intervals of time 107 between divergence events interpreted as transmission events. This situation can be 108 translated in terms of survival analysis, which models rates of event occurrence, by 109 considering divergence as the event of interest and by treating branch lengths as time110 to-event intervals (Fig. 1c, d). Phylogenetic survival analysis was devised by E. Paradis 111 and applied to detecting temporal variations in the divergence rate of tanagers ${ }^{12}$ or 112 fishes ${ }^{15}$, but it has not been applied to pathogens so far ${ }^{13,16,17}$.

113 To quantify the effect of non-pharmaceutical interventions on the transmission 114 rate of COVID-19, we adapted the original model in the Paradis study ${ }^{12}$ to account for 115 the specific setting of viral phylodynamics (see Methods). Hereafter, we refer to the 116 modified model as phylodynamic survival analysis. In survival analysis terms, we 117 interpret internal branches of the phylogeny (those that end with a transmission event) 118 as time-to-event intervals and terminal branches (those that end with a sampling event) 119 as censored intervals (Fig. 1c; see Methods).

120 The predictors of interest in our setting, namely, the non-pharmaceutical 121 interventions, vary both through time and across lineages depending on their geographic location. To model this, we assigned each divergence event (and 123 subsequent branch) to a country using maximum-likelihood ancestral state 124 reconstruction. Each assigned branch was then associated with the set of non125 pharmaceutical interventions that were active or not in the country during the interval 126 spanned by the branch. Intervals containing a change of intervention were split into 127 subintervals ${ }^{18}$. These (sub)intervals were the final observations (statistical units) used in 
128 the survival models. Models were adjusted for the hierarchical dependency structure 129 introduced by interval splits and country assignations (see Methods).

130 Phylodynamic survival models estimate variations of the reproduction number

131 The evolution of lineages in a dated viral phylogeny can be described as a birth-death 132 process with a divergence (or birth) rate $\lambda$ and an extinction (or death) rate $\mu^{19}$. In a 133 phylodynamic context, the effective reproduction number $R_{t}$ equals the ratio of the 134 divergence and extinction rates ${ }^{19}$. Coefficients of phylodynamic survival models (the so135 called hazard ratios of divergence; see Methods) act as multiplicative factors of the 136 divergence rate $\lambda$, independent of the true value of $\lambda$ which needs not be specified nor 137 evaluated. As $R_{t}=\lambda / \mu$, multiplying $\lambda$ by a coefficient also multiplies $R_{t}$, independent of 138 the true value of $\mu$. Thus, coefficients of phylodynamic survival models estimate 139 variations of $R_{t}$ in response to predictor variables without requiring external knowledge 140 or assumptions about $\lambda$ and $\mu$. 
a

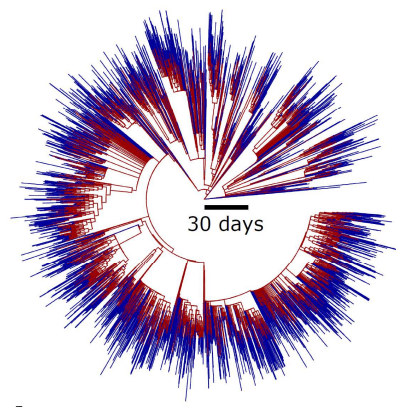

b

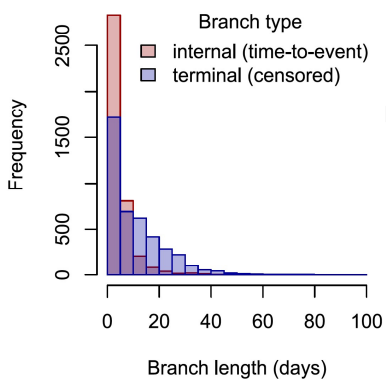

e

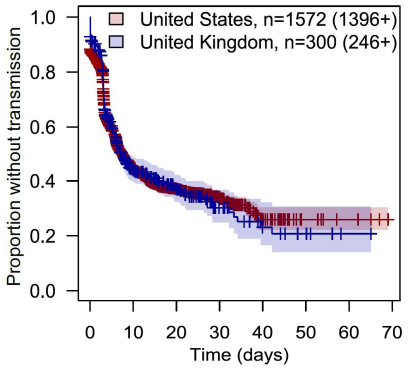

C
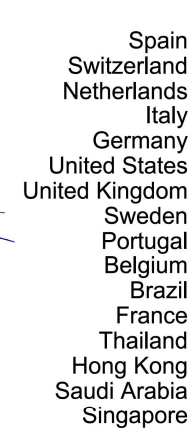

Spain -

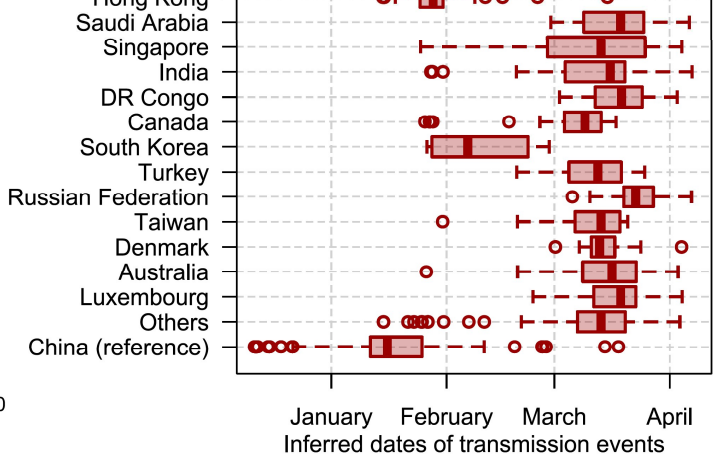

$\mathbf{f}$

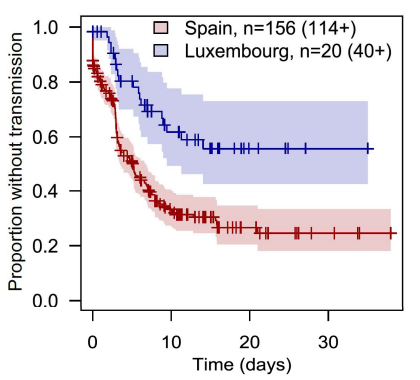

d

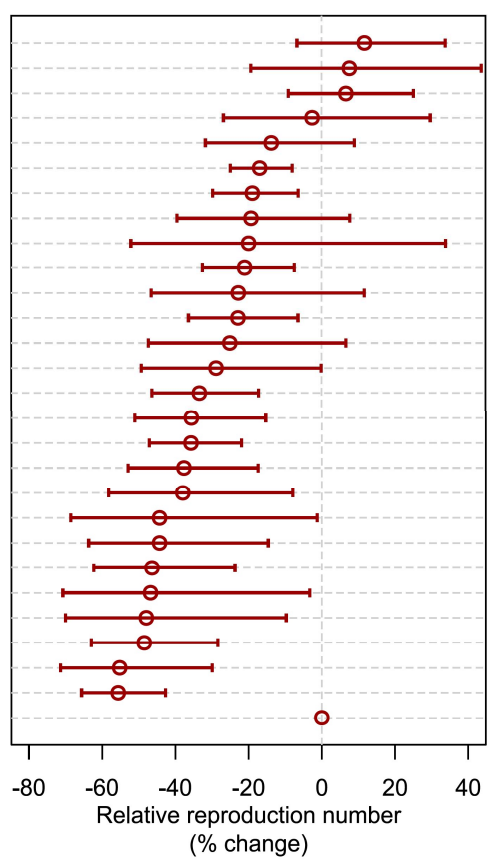

h
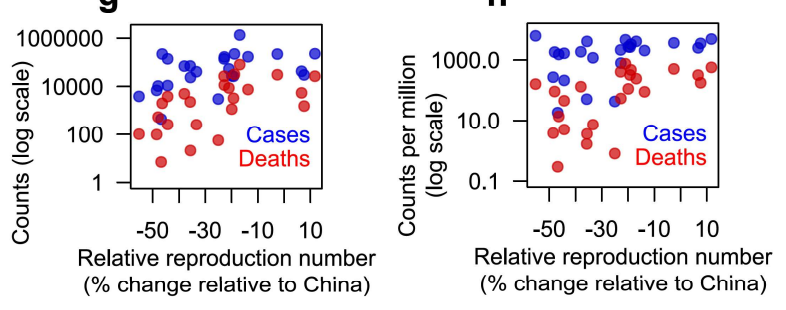

Figure 2. Timing and reproduction numbers of the COVID-19 epidemic in 74 countries based on a dated phylogeny. a, Dated phylogeny of 5,198 SARS-CoV-2 genomes where internal (time-to-event) and terminal (time-to-censoring) branches are colored red and blue, respectively. b, Histogram of internal and terminal branch lengths. c, Box-and-whisker plots of the distribution over time of the inferred transmission events in each country, where boxes denote interquartile range (IQR) and median, whiskers extend to dates at most 1.5x the IQR away from the median date, and circle marks denote dates farther than 1.5 IQR from the median date. d, Point estimates and 95\% confidence intervals of the relative effective reproduction number, expressed as percent changes relative to China, in 27 countries with $\geq 10$ assigned transmission events. Countries with $<10$ assigned transmission events $(n=32)$ were pooled into the 'Others' category. e, f, Representative Kaplan-Meier survival curves of the probability of transmission through time in countries with comparable (e) or contrasting (f) transmission rates. ' + ' marks denote censoring events. Numbers denote counts of internal branches and, in brackets, terminal branches. $\mathbf{g}, \mathbf{h}$, Scatter plots showing correlations between the relative reproduction number and the reported cumulative numbers of COVID-19 cases (blue marks) and deaths (red marks) per country up to May 12, 2020 (53), in absolute values (g) and per million inhabitants $(\mathbf{h})$. 
161 We assembled a composite dataset by combining a dated phylogeny of SARS-CoV-2 162 (Fig. 2a), publicly available from Nextstrain ${ }^{20}$ and built from the GISAID initiative data ${ }^{21}$, with a detailed timeline of non-pharmaceutical interventions available from the Oxford COVID-19 Government Response Tracker (OxCGRT) ${ }^{22}$. Extended Data Fig. 1 shows a flowchart outlining the data sources, sample sizes and selection steps of the study. Phylogenetic and intervention data covered the early phase of the epidemic up to May 4, 2020.

The 5,198 SARS-CoV-2 genomes used to reconstruct the dated phylogeny were collected from 74 countries. Detailed per-country data including sample sizes are shown in Extended Data Table 1. Among the 10,394 branches in the phylogeny, 2,162 branches $(20.8 \%)$ could not be assigned to a country with $>95 \%$ confidence and were excluded, also reducing the number of represented countries from 74 to 59 (Extended Data Fig. 1; a comparison of included and excluded branches is shown in Extended Data Fig. 2). The remaining 4,025 internal branches had a mean time-to-event (delay between transmission events) of 4.4 days (Fig. 2b). These data were congruent with previous estimates of the mean serial interval of COVID-19 ranging from 3.1 days to 7.5 days $^{23}$. The 4,207 terminal branches had a mean time-to-censoring (delay from infection to detection) of 10.6 days (Fig. 2a, b). This pattern of longer terminal vs. internal branches is typical of a viral population in fast expansion ${ }^{11}$.

We compared the timing and dynamics of COVID-19 spread in countries

represented in our dataset (Fig. 2c, d), pooling countries with $<10$ assigned transmission events into an 'others' category. The estimated date of the first local transmission event in each country showed good concordance with the reported dates of the epidemic onset (Pearson correlation $=0.84$; Extended Data Fig. 3). The relative effective reproduction number $R_{t}$ per country, taking China as reference, ranged from $55.6 \%(95 \% \mathrm{Cl},-71.4 \%$ to $-29.9 \%)$ in Luxembourg to $+11.7 \%(95 \% \mathrm{Cl},-6.7 \%$ to $+33.8 \%)$ in Spain (Fig. 2c). Notice that these estimates are averages over variations of $R_{t}$ through time in each country, up to May 4, 2020. Exemplary survival curves of 
189 transmission events are shown in Fig. 2e, f. Relative $R_{t}$ 's are not expected to 190 necessarily correlate with the reported counts of COVID-19 cases across all countries 191 due to the confounding effects of population sizes, case detection policies and the 192 number of genomes included. Nevertheless, the relative $R_{t}$ 's across countries 193 substantially correlated with the reported cumulative counts up to May 12 (Fig. $2 \mathrm{~g}, \mathrm{~h}$ ), 194 including COVID-19 cases (Pearson correlation with log-transformed counts, 0.46, 95\% $195 \mathrm{Cl}, 0.07$ to 0.73 ), deaths (correlation $0.59,95 \% \mathrm{Cl}, 0.24$ to 0.80 ), cases per million 196 inhabitants (correlation $0.39,95 \% \mathrm{Cl},-0.01$ to 0.69 ) and deaths per million inhabitants 197 (correlation $0.56,95 \% \mathrm{Cl}, 0.21$ to 0.79 ). 
199 Table 1. Selected large-scale non-pharmaceutical interventions against COVID-19.

\begin{tabular}{|c|c|c|}
\hline Non-pharmaceutical intervention & OxCGRT identifier & Definition \\
\hline Information campaign & $\mathrm{H} 1$ & $\begin{array}{l}\text { Coordinated public information } \\
\text { campaign across traditional and social } \\
\text { media }\end{array}$ \\
\hline Restrict international travel & $\mathrm{C} 8$ & $\begin{array}{c}\begin{array}{c}\text { Ban or quarantine arrivals from high-risk } \\
\text { regions }\end{array}\end{array}$ \\
\hline Education lockdown & $\mathrm{C} 1$ & $\begin{array}{l}\text { Require closing for some or all } \\
\text { education levels or categories, e.g., high } \\
\text { schools, public schools, universities }\end{array}$ \\
\hline Cancel public events & C3 & Require cancelling of all public events \\
\hline Restrict gatherings $>100$ pers. & $\mathrm{C} 4$ & Prohibit gatherings over 100 persons \\
\hline Close workplaces & $\mathrm{C} 2$ & $\begin{array}{l}\text { Require closing or working from home } \\
\text { for some or all non-essential sectors or } \\
\text { categories of workers }\end{array}$ \\
\hline Restrict internal movements & $\mathrm{C} 7$ & $\begin{array}{l}\text { Require closing routes or prohibit most } \\
\text { citizens from using them }\end{array}$ \\
\hline Close public transport & $\mathrm{C} 5$ & $\begin{array}{l}\text { Require closing of public transport or } \\
\text { prohibit most citizens from using it }\end{array}$ \\
\hline Home containment & $\mathrm{C} 6$ & $\begin{array}{l}\text { Require not leaving house with or } \\
\text { without exceptions for daily exercise, } \\
\text { grocery shopping and essential trips }\end{array}$ \\
\hline
\end{tabular}

NOTE. OxCGRT, Oxford COVID-19 Government Response Tracker initiative, www.bsg.ox.ac.uk/covidtracker 

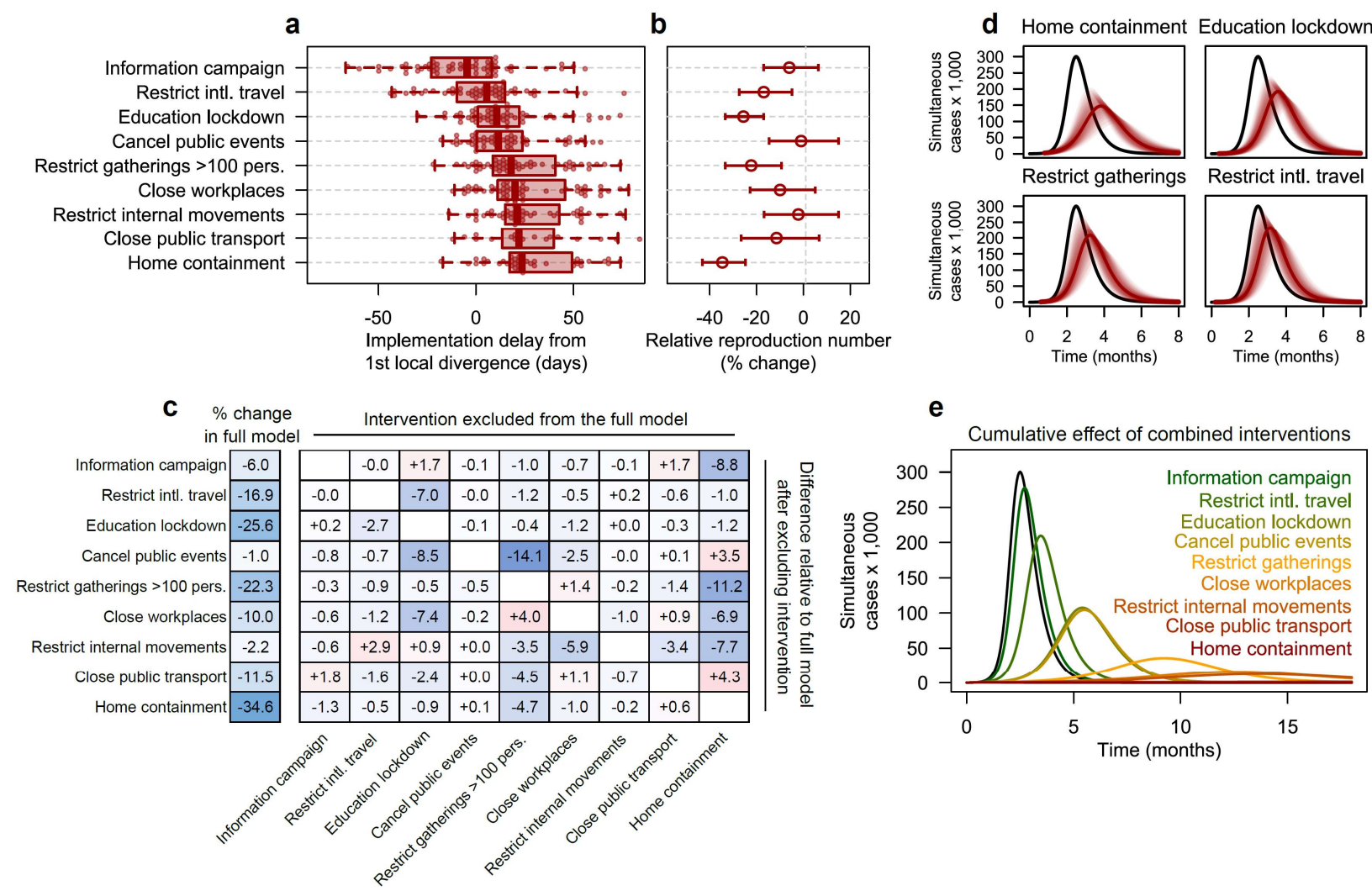

Figure 3. Non-pharmaceutical interventions variably reduce the reproduction number of CoVID-19. Data derive from the phylogenetic survival analysis of 4,191 internal and 4,019 terminal branches of a dated phylogeny of SARS-CoV-2 genomes, combined with a chronology of interventions in 57 countries. a, Box-and-whisker plots of the delay between the 1st SARSCoV-2 divergence event and the intervention. Plot interpretation is similar with Fig. 2c. b. Point estimates and $95 \%$ confidence intervals of the independent $\%$ change of the effective reproduction number predicted by each intervention in a multivariable, mixed-effect phylogenetic survival model adjusted for between-country variations. c, Matrix of pairwise interactions between the interventions (in rows) estimated using 9 multivariable models (in columns), where each model ignores exactly one intervention. Negative (positive) differences in blue (red) denote a stronger (lesser) predicted effect of the intervention in row when ignoring the intervention in column. d, e, Simulated impact of interventions implemented independently (d) or in sequential combination (e) on the count of simultaneous cases in an idealized population of 1 million susceptible individuals using compartmental SIR models with a basic reproduction number $R_{0}=$ 3 (black lines) and a mean infectious period of 2 weeks. Shaded areas in (d) denote $95 \%$ confidence bands. 
219 The implementation and release dates of large-scale non-pharmaceutical interventions 220 against COVID-19 were available for 57 countries out of the 59 represented in the dated 221 phylogeny. Definitions of the selected interventions are shown in Table 1. Branches 222 assigned to countries with missing intervention data, namely, Latvia and Senegal, were 223 excluded from further analysis ( $n=22 / 8,262$ ( $0.3 \%)$; see Extended Data Fig. 1 ). Up to 224 May 4, 2020, the interventions most universally implemented were information 225 campaigns, restrictions on international travel and education lockdown ( $>95 \%$ of 226 countries) (Extended Data Fig. 4). The least frequent were the closure of public transportation (35\%) and home containment (72\%). Public information campaigns came first and home containment came last (median delay across countries, 5 days before and 24 days after the first local transmission event, respectively; Fig. 3a). Survival curves for each intervention are shown in Extended Data Fig. 5. Most interventions were implemented in combination and accumulated over time rather than replacing each other (Extended Data Fig. 4; median delays between interventions are shown in Extended Data Fig. 6; correlations in Extended Data Fig. 7; and a detailed timeline of interventions in Extended Data Table 2). However, we observed a substantial heterogeneity of intervention timing across the 57 countries (Fig. 3a), suggesting that individual intervention effects can be discriminated by multivariable analysis given the large sample size ( $n=8,210$ subintervals).

A multivariable phylogenetic survival model, including the 9 interventions and controlling for between-country $R_{t}$ variations (see Methods), showed a strong fit to the data (likelihood-ratio test compared to the null model, $\mathrm{P}<10^{-196}$ ). In this model, the interventions most strongly and independently associated with a reduction of the effective reproduction number $R_{t}$ of SARS-CoV-2 were home containment $\left(R_{t}\right.$ percent change, $-34.6 \%, 95 \% \mathrm{Cl},-43.2$ to $-24.7 \%)$, education lockdown $(-25.6 \%, 95 \% \mathrm{Cl},-33.4$ to $-16.9 \%)$, restricting gatherings $(-22.3 \%, 95 \% \mathrm{Cl},-33.4$ to $-9.4 \%)$ and international travel $(-16.9 \%, 95 \% \mathrm{Cl},-27.5$ to $-4.8 \%)$. We failed to detect a substantial impact of other interventions, namely information campaigns, cancelling public events, closing 
247 workplaces, restricting internal movement, and closing public transportation (Fig. 3b).

248 Based on coefficient estimates, all interventions were independently predicted to reduce

$249 R_{t}$ (even by a negligible amount), in line with the intuition that no intervention should

250 accelerate the epidemic. Contrasting with previous approaches that constrained

251 coefficients ${ }^{5}$, this intuition was not enforced a priori in our multivariable model, in which

252 positive coefficients (increasing $R_{t}$ ) might have arisen due to noise or collinearity

253 between interventions. The absence of unexpectedly positive coefficients suggests that

254 our model correctly captured the epidemic slowdown that accompanied the

255 accumulation of interventions.

256 Estimated intervention effects are robust to time-dependent confounders and 257 collinearity

258 A reduction of $R_{t}$ through time, independent of the implementation of interventions, 259 might lead to overestimate their effect in our model. Several potential confounders might 260 reduce $R_{t}$ through time but cannot be precisely estimated and included as control covariates. These included the progressive acquisition of herd immunity, the so-called artificial diversification slowdown possibly caused by incomplete sampling, and timedependent variations of the sampling effort (see Methods). To quantify this potential time-dependent bias, we constructed an additional model including the age of each branch as a covariate (Extended Data Table 1). The coefficients in this time-adjusted model only differed by small amounts compared to the base model. Moreover, the ranking by effectiveness of the major interventions remained unchanged, indicating that our estimates were robust to time-dependent confounders.

We also quantified the sensitivity of the estimated intervention effects to the inclusion of other interventions (collinearity) by excluding interventions one by one in 9 additional models (Fig. 3c). This pairwise interaction analysis confirmed that most of the estimated effects were strongly independent. Residual interferences were found for gathering restrictions, whose full-model effect of $-22.3 \%$ was reinforced to $-33.5 \%$ when ignoring home containment; and for cancelling public events, whose full-model effect of $-0.97 \%$ was reinforced to $-15.1 \%$ when ignoring gathering restrictions. These residual 
interferences make epidemiological sense because home containment prevents gatherings and gathering restrictions also prohibit public events. Overall, the absence of strong interferences indicated that our multivariable model reasonably captured the independent, cumulative effect of interventions, enabling ranking their impact on COVID-19 spread.

Table 2. Predicted reduction of the COVID-19 effective reproduction number under increasingly stringent combinations of non-pharmaceutical interventions.

\begin{tabular}{lcccccc}
\hline & & \multicolumn{5}{c}{ Probability of reducing $\boldsymbol{R}_{\boldsymbol{t}}$ below $\mathbf{1}$} \\
\cline { 3 - 7 } Accumulated interventions & $\begin{array}{c}\text { Relative } \boldsymbol{R}_{\boldsymbol{t}} \\
\text { (cumulative \% change) }\end{array}$ & $\boldsymbol{R}_{\boldsymbol{0}}=\mathbf{1 . 5}$ & $\boldsymbol{R}_{0}=\mathbf{2 . 0}$ & $\boldsymbol{R}_{\boldsymbol{0}}=\mathbf{2 . 5}$ & $\boldsymbol{R}_{\boldsymbol{0}}=\mathbf{3 . 0}$ & $\boldsymbol{R}_{\boldsymbol{0}}=\mathbf{3 . 5}$ \\
\hline Information campaign & $-6.0(-17.0$ to +6.5$)$ & $<0.01$ & $<0.01$ & $<0.01$ & $<0.01$ & $<0.01$ \\
+ Restrict intl. travel & $-21.9(-35.0$ to -6.1$)$ & 0.05 & $<0.01$ & $<0.01$ & $<0.01$ & $<0.01$ \\
+ Education lockdown & $-41.9(-52.5$ to -29.0$)$ & 0.91 & 0.07 & $<0.01$ & $<0.01$ & $<0.01$ \\
+ Cancel public events & $-42.5(-54.0$ to -28.1$)$ & 0.90 & 0.11 & $<0.01$ & $<0.01$ & $<0.01$ \\
+ Restrict gatherings $>100$ pers. & $-55.3(-63.4$ to -45.5$)$ & 1.00 & 0.86 & 0.14 & $<0.01$ & $<0.01$ \\
+ Close workplaces & $-59.7(-67.6$ to -50.0$)$ & 1.00 & 0.98 & 0.48 & 0.04 & $<0.01$ \\
+ Restrict internal movements & $-60.6(-67.9$ to -51.6$)$ & 1.00 & 0.99 & 0.56 & 0.06 & $<0.01$ \\
+ Close public transport & $-65.1(-72.6$ to -55.7$)$ & 1.00 & 1.00 & 0.87 & 0.36 & 0.05 \\
+ Home containment & $-77.2(-81.5$ to -71.9$)$ & 1.00 & 1.00 & 1.00 & 1.00 & 0.98 \\
\hline
\end{tabular}

\section{Simulating intervention effectiveness in an idealized population}

To facilitate the interpretation of our estimates of the effectiveness of interventions against COVID-19, we simulated each intervention's impact on the peak number of cases, whose reduction is critical to prevent overwhelming the healthcare system (Fig. 3d and Extended Data Fig. 8). We used compartmental Susceptible-Infected-Recovered (SIR) models with a basic reproduction number $R_{0}=3$ and a mean infectious period of 2 weeks based on previous estimates ${ }^{24,25}$, in an idealized population of 1 million susceptible individuals (see Methods). In each model, we simulated the implementation of a single intervention at a date chosen to reflect the median delay across countries (Fig. 3a) relative to the epidemic onset (see Methods). On implementation date, the 
293 effective reproduction number was immediately reduced according to the estimated 294 intervention's effect shown in Fig. 3b.

In this idealized setting, home containment, independent of all other restrictions, 296 only halved the peak number of cases from $3.0 \times 10^{5}$ to $1.5 \times 10^{5}\left(95 \% \mathrm{Cl}, 1.0 \times 10^{5}\right.$ to $\left.2972.0 \times 10^{5}\right)$ (Fig. 3d). However, a realistic implementation of home containment also 298 implies other restrictions including, at least, restrictions on movements, gatherings, and 299 public events. This combination resulted in a relative $R_{t}$ of $-50.8 \%(95 \% \mathrm{Cl},-59.4 \%$ to $30040.2 \%)$ and a 5 -fold reduction of the peak number of cases to $6.0 \times 10^{4}\left(95 \% \mathrm{Cl}, 1.9 \times 10^{4}\right.$ 301 to $1.2 \times 10^{5}$ ). Nevertheless, if $R_{0}=3$ then a $50 \%$ reduction is still insufficient to reduce $R_{t}$ 302 below 1 and stop the epidemic. This suggests that even when considering the most 303 stringent interventions, combinations may be required. To further examine this issue, we 304 estimated the effect of accumulating interventions by their average chronological order 305 shown in Fig. 3a, from information campaigns alone to all interventions combined 306 including home containment (Fig. 3e). Strikingly, only the combination of all 307 308 309 interventions completely stopped the epidemic under our assumed value of $R_{0}$. To estimate the effectiveness of combined interventions in other epidemic settings, we computed the probabilities of reducing $R_{t}$ below 1 for values of $R_{0}$ ranging from 1.5 to 3.5 (Table 2; see Methods). The same probabilities for individual interventions are presented in Table S2, showing that no single intervention would stop the epidemic if $R_{0} \geq 2$. These results may help inform decisions on the appropriate stringency of the 


\section{Discussion}

316 We present a phylodynamic analysis of how the divergence rate and reproduction

317 number of SARS-CoV-2 varies in response to large-scale non-pharmaceutical

318 interventions in 57 countries. Our results suggest that no single intervention, including

319 home containment, is sufficient on its own to stop the epidemic $\left(R_{t}<1\right)$. Increasingly

320 stringent combinations of interventions may be required depending on the effective

321 reproduction number.

322 Home containment was repeatedly estimated to be the most effective response 323 in epidemiological studies from China ${ }^{26}$, France ${ }^{27}$, the $\mathrm{UK}^{28}$, and Europe ${ }^{5}$. Other studies 324 modelled the additional (or residual) reduction of $R_{t}$ by an intervention after taking into 325 account those previously implemented ${ }^{4,6,10}$. Possibly because home containment was 326 the last implemented intervention in many countries (Fig. 2a), these studies reported a 327 weaker or even negligible additional effect compared to earlier interventions. In our 328 study, home containment, even when implemented last, had the strongest independent 329 impact on epidemic spread $\left(R_{t}\right.$ percent change, $\left.-34.6 \%\right)$, which was further amplified ($33050.8 \%$ ) when taking into account implicit restrictions on movements, gatherings and 331 public events.

We found that education lockdown substantially decreased COVID-19 spread $\left(R_{t}\right.$ percent change, $-25.6 \%)$. Contrasting with home containment, the effectiveness of education lockdown has been more hotly debated. This intervention ranked among the most effective ones in two international studies ${ }^{4,6}$ but had virtually no effect on transmission in other reports from Europe ${ }^{5,10}$. Young children have been estimated to be poor spreaders of COVID-19 and less susceptible than adults to develop disease after an infectious contact, counteracting the effect of their higher contact rate ${ }^{29,30}$. However, the relative susceptibility to infection was shown to increase sharply between 15 and 25 years, suggesting that older students might be more involved in epidemic spread ${ }^{30}$. Importantly, we could not differentiate the effect of closing schools and universities because both closures coincided in all countries. Thus, our finding that education lockdowns reduce COVID-19 transmission might be driven by contact rate reductions in 
older students rather than in children, as hypothesized elsewhere ${ }^{4}$. This raises the question of whether education lockdown should be adapted to age groups, considering that: (1), education lockdown had a sizeable impact on COVID-19 transmission in our study and others ${ }^{4,6} ;(2)$, this impact might be preferentially driven by older students ${ }^{29,30}$; and (3) autonomous distance learning might be more effective in university students ${ }^{31}$ compared to younger children who require parental presence and supervision following school closure, possibly widening the gap for children from under-resourced backgrounds ${ }^{32}$. Based on these elements, we speculate that closing universities, but not elementary schools, might strike the right balance between efficacy and social impact.

Restrictions on gatherings of $>100$ persons appeared more effective than cancelling public events ( $R_{t}$ percent changes, $-22.3 \%$ vs. $-1.0 \%$, respectively) in our phylodynamic model, in line with previous results from epidemiological models ${ }^{4}$. Notwithstanding that gathering restrictions prohibit public events, possibly causing interferences between estimates (Fig. 3c), this finding is intriguing. Indeed, several public events resulted in large case clusters, the so-called superspreading events, that triggered epidemic bursts in France ${ }^{33}$, South $\mathrm{Korea}^{34}$ or the U.S. ${ }^{35}$. A plausible explanation for not detecting the effectiveness of cancelling public events is that datadriven models, including ours, better capture the cumulative effect of more frequent events such as gatherings than the massive effect of much rarer events such as superspreading public events. This bias towards ignoring the so-called 'Black Swan' exceptional events ${ }^{36}$ suggests that our findings (and others'4) regarding restrictions on public events should not be interpreted as an encouragement to relax these restrictions but as a potential limit of modelling approaches (but see ${ }^{37}$ ).

There are other limitations to our study, including its retrospective design. Similar to previous work ${ }^{6}$, we did not consider targeted non-pharmaceutical interventions that are difficult to date and quantify, such as contact tracing or case isolation policies. Data were analyzed at the national level, although much virus transmission was often concentrated in specific areas and some non-pharmaceutical interventions were implemented at the sub-national level ${ }^{38}$. Our phylogeographic inferences did not consider the travel history of patients, whose inclusion in Bayesian models was recently 
374 shown to alleviate sampling bias ${ }^{39}$. From a statistical standpoint, the interval lengths in 375 the dated phylogeny were treated as fixed quantities in the survival models. Ignoring the 376 uncertainty of the estimated lengths might underestimate the width of confidence 377 intervals, although this is unlikely to have biased the pointwise estimates and the 378 ranking of interventions' effects. The number of genomes included by country did not 379 necessarily reflect the true number of cases, which might have influenced country 380 comparison results in Fig. 2, but not intervention effectiveness models in Fig. 3 which 381 were adjusted for between-country variations of $R_{t}$. Finally, our estimates represent 382 averages over many countries with different epidemiological contexts, healthcare 383 systems, cultural behaviors and nuances in intervention implementation details and 384 population compliance. This global approach, similar to previous work ${ }^{4,6}$, facilitates 385 unifying the interpretation of intervention effectiveness, but this interpretation still needs 386 to be adjusted to local contexts by policy makers.

387 Beyond the insights gained into the impact of interventions against COVID-19, 388 our findings highlight how phylodynamic survival analysis can help leverage pathogen sequence data to estimate epidemiological parameters. Contrasting with the Bayesian approaches adopted by most, if not all, previous assessments of intervention effectiveness ${ }^{4,5,8}$, phylodynamic survival analysis does not require any quantitative prior 393 assumption or constraint on model parameters. The method should also be simple to implement and extend by leveraging the extensive software arsenal of survival modelling. Phylodynamic survival analysis may complement epidemiological models as 396 pathogen sequences accumulate, allowing to address increasingly complex questions relevant to public health strategies. 
Definitions and chronology of non-pharmaceutical interventions

The nature, stringency and timing of non-pharmaceutical interventions against COVID19 have been collected and aggregated daily since January 1, 2020 by the Oxford COVID-19 Government Response Tracker initiative of the Blavatnik School of Government, UK ${ }^{4,22}$. As of May 12, 2020, the interventions are grouped into three categories, namely: closures and containment (8 indicators), economic measures (4 indicators) and health measures (5 indicators). Indicators use 2- to 4-level ordinal scales to represent each intervention's stringency, and an additional flag indicating whether the intervention is localized or general. Details of the coding methods for indicators can be found $\mathrm{in}^{40}$. We focused on large-scale interventions against transmission that did not target specific patients (for instance, we did not consider contact tracing) and we excluded economic and health interventions except for information campaigns. This rationale led to the selection of the 9 indicators shown in Table 1. To facilitate interpretation while constraining model complexity, the ordinal-scale indicators in OxCGRT data were recoded as binary variables in which we only considered government requirements (as opposed to recommendations) where applicable. We did not distinguish between localized and nation-wide interventions because localized interventions, especially in larger countries, targeted the identified epidemic hotspots. As the data did not allow to differentiate closures of schools and universities, we use the term 'education lockdown' (as opposed to 'school closure' in ${ }^{22}$ ) to avoid misinterpretation regarding the education levels concerned.

\section{Phylodynamic survival analysis in measurably evolving populations}

The original phylogenetic survival model in ${ }^{12}$ and its later extensions ${ }^{41}$ considered intervals backward in time, from the tips to the root of the tree, and were restricted to trees with all tips sampled at the same date relative to the root (ultrametric trees). Censored intervals (intervals that do not end with an event) in ${ }^{12}$ were used to represent lineages with known sampling date but unknown age. In contrast, viral samples in 
426 ongoing epidemics such as COVID-19 are typically collected through time. A significant 427 evolution of the viruses during the sampling period violates the ultrametric assumption. 428 To handle phylogenies of these so-called measurably evolving populations ${ }^{42}$, we 429 propose a different interpretation of censoring compared to ${ }^{12}$. Going forward in time, the 430 internal branches of a tree connect two divergence events while terminal branches, 431 those that end with a tip, connect a divergence event and a sampling event (Fig. 1b). 432 Thus, we considered internal branches as time-to-event intervals and terminal branches 433 as censored intervals representing the minimal duration during which no divergence 434 occurred (Fig. 1c).

\section{SARS-CoV-2 phylogenetic data}

SARS-CoV-2 genome sequences have been continuously submitted to the Global Initiative on Sharing All Influenza Data (GISAID) by laboratories worldwide ${ }^{21}$. To circumvent the computational limits of phylogeny reconstruction and time calibration techniques, the sequences of the GISAID database are subsampled before analysis by the Nextstrain initiative, using a balanced subsampling scheme through time and space $^{20,43}$. Phylogenetic reconstruction uses maximum-likelihood phylogenetic inference based on IQ-TREE ${ }^{44}$ and time-calibration uses TreeTime ${ }^{45}$. See ${ }^{46}$ for further details on the Nextstrain bioinformatics pipeline. A dated phylogeny of 5,211 SARS-CoV-2 genomes, along with sampling dates and locations, was retrieved from nextstrain.org/ncov on May 12, 2020. Genomes of non-human origin $(n=13)$ were discarded from analysis. Polytomies (unresolved divergences represented as a node with $>2$ descendants) were resolved as branches with an arbitrarily small length of 1 hour, as recommended for adjustment of zero-length risk intervals in Cox regression ${ }^{47}$. Of note, excluding these zero-length branches would bias the analysis by underestimating the number of divergence events in specific regions of the phylogeny. Maximum-likelihood ancestral state reconstruction was used to assign internal nodes of the phylogeny to countries in a probabilistic fashion, taking the tree shape and sampling locations as input data ${ }^{48}$. To prepare data for survival analysis, we decomposed the branches of the dated phylogeny into a set of time-to-event and time-to-censoring 
455 intervals (Fig. 1c). Intervals were assigned to the most likely country at the origin of the 456 branch when this country's likelihood was $>0.95$. Intervals in which no country reached 457 a likelihood of 0.95 were excluded from further analysis (Extended Data Figs. 1, 2). 458 Finally, intervals during which a change of intervention occurred were split into sub459 intervals, such that all covariates, including the country and interventions, were held 460 constant within each sub-interval and only the last subinterval of an internal branch was 461 treated as a time-to-event interval. This interval-splitting approach is consistent with an 462 interpretation of interventions as external time-dependent covariates ${ }^{18}$, which are not dependent on the event under study, namely, the viral divergence event.

\section{Mixed-effect Cox proportional hazard models}

465 Variations of the divergence rate $\lambda$ in response to non-pharmaceutical interventions 466 were modelled using mixed-effect Cox proportional hazard regression (reviewed in ${ }^{49}$ ). Models treated the country and phylogenetic branch as random effects to account for non-independence between sub-intervals of the same branch and between branches assigned to the same country. The predictors of interest were not heritable traits of SARS-CoV-2, thus, phylogenetic autocorrelation between intervals was not corrected for. Time-to-event data were visualized using Kaplan-Meier curves with 95\% confidence intervals. The regression models had the form

$$
\lambda_{i}(t)=\lambda_{0}(t) \cdot \exp \left(X_{i} \cdot \beta+\alpha_{j}+\gamma_{k}\right)
$$

where $\lambda_{i}(t)$ is the hazard function (here, the divergence rate) at time $t$ for the $i$ th observation, $\lambda_{0}(t)$ is the baseline hazard function, which is neither specified or explicitly evaluated, $X_{i}$ is the set of predictors of the $i$ th observation (the binary vector of active non-pharmaceutical interventions), $\beta$ is the vector of fixed-effect coefficients, $\alpha_{j}$ is the random intercept associated with the $j$ th phylogenetic branch and $\gamma_{k}$ is the random intercept associated with the $k$ th country. Country comparison models (Fig. 2d), in which the country was the only predictor and branches were not divided into subintervals, did not include random intercepts. Raw model coefficients (the log-hazard ratios) additively shift the logarithm of the divergence rate $\lambda$. Exponentiated coefficients 
$483 \exp \beta$ (the hazard ratios) are multiplicative factors (fold-changes) of the divergence rate. 484 To ease interpretation, hazard ratios were reported as percentage changes of the 485 divergence rate or, equivalently, of the effective reproduction number $R_{t}$, equal to $486(\exp \beta-1) \times 100$. Analyses were conducted using R 3.6.1 (the R Foundation for 487 Statistical Computing, Vienna, Austria) with additional packages ape, survival and $488 \quad$ coxme.

\section{Estimating the effect of combined interventions}

490 Pointwise estimates and confidence intervals of combined interventions were estimated by adding individual coefficients and their variance-covariances. Cox regression coefficients have approximately normal distribution with mean vector $m$ and variancecovariance matrix $V$, estimated from the inverse Hessian matrix of the likelihood function evaluated at $m$. From well-known properties of the normal distribution, the distribution of a sum of normal deviates is normal with mean equal to the sum of the means and variance equal to the sum of the variance-covariance matrix of the deviates. Thus, the coefficient corresponding to a sum of coefficients with mean $m$ and variance $V$ has mean $\sum m$ and variance $\sum V$, from which we derive the point estimates and confidence intervals of a combination of predictors. Importantly, summing over the covariances captures the correlation between coefficients when estimating the uncertainty of the combined coefficient.

\section{Probability of stopping an epidemic}

A central question regarding the effectiveness of interventions or combinations thereof approximately normal distribution with mean $\beta$ and variance $\sigma^{2}$, written $\hat{\beta} \sim N\left(\beta, \sigma^{2}\right)$. For some fixed value of $R_{0}$, the estimated post-intervention reproduction number $\widehat{R_{t}}=$ $R_{0} \cdot \exp \hat{\beta}$. The probability $p$ that $\widehat{R_{t}}<1$ is $\int_{-\infty}^{1} d\left(\widehat{R_{t}}\right) \mathrm{d} \widehat{R_{t}}$ where $d$ denotes the probability density function. To solve the integral, remark that $\log \widehat{R_{t}}=\log R_{0}+\hat{\beta} \sim N\left(\log R_{0}+\right.$ 
$\left.510 \hat{\beta}, \sigma^{2}\right)$. Using a change of variables in the integral and noting that $\log 1=0$, we obtain

511 the closed-form solution

$$
p=\int_{-\infty}^{0} d\left(\log \widehat{R_{t}}\right) d \log \widehat{R_{t}}=\Phi\left(0 \mid \log R_{0}+\hat{\beta}, \sigma^{2}\right),
$$

513 where $\Phi$ is the cumulative density function of the normal distribution with mean $\log R_{0}+$ $514 \hat{\beta}$ and variance $\sigma^{2}$. By integrating over the coefficient distribution, this method explicitly 515 considers the estimation uncertainty of $\hat{\beta}$ when estimating $p$.

\section{Potential time-dependent confounders}

517 Time-dependent phylodynamic survival analysis assumes that variations of branch 518 lengths though time directly reflect variations of the divergence rate, which implies that 519 branch lengths are conditionally independent of time given the divergence rate. When the phylogeny is reconstructed from a fraction of the individuals, as is the case in virtually all phylodynamic studies including ours, this conditional independence assumption can be violated. This is because incomplete sampling increases the length of more recent branches relative to older branches ${ }^{50}$, an effect called the diversification slowdown ${ }^{51,52}$. Noteworthy, this effect can be counteracted by a high extinction rate ${ }^{17,50}$, which is expected in our setting and mimicks an acceleration of diversification. Moreover, whether the diversification slowdown should be interpreted as a pure artifact has been controversial ${ }^{52,53}$. Notwithstanding, we considered incomplete sampling as a potential source of bias in our analyses because a diversification slowdown might lead to an overestimation of the effect of non-pharmaceutical interventions. Additionally, the selection procedure used by Nextstrain to collect genomes included in the dated phylogeny possibly amplified the diversification slowdown by using a higher sampling fraction in earlier phases of the epidemic ${ }^{43}$. To verify whether the conclusions of our models were robust to this potential bias, we built an additional multivariable model including the estimated date of each divergence event (the origin of the branch) as a covariate. The possible relation between time and the divergence rate is expectedly non-linear ${ }^{50}$ and coefficient variations resulting from controlling for time were moderate 
537 (Extended Data Table 1), thus, we refrained from including a time covariate in the 538 reported regression models as this might lead to overcontrol. Further research is 539 warranted to identify an optimal function of time that might be included as a covariate in 540 phylodynamic survival models to control for sources of diversification slowdown.

\section{$541 \quad$ Compartmental epidemiological models}

542 Epidemic dynamics can be described by partitioning a population of size $N$ into three compartments, the susceptible hosts $S$, the infected hosts $I$, and the recovered hosts $R$.

$544 \quad$ The infection rate $b$ governs the transitions from $S$ to $I$ and the recovery rate $g$ governs $545 \quad$ the transitions from $I$ to $R$ (we avoid the standard notation $\beta$ and $\gamma$ for infection and 546 recovery rates to prevent confusion with Cox model parameters). The SIR model 547 describes the transition rates between compartments as a set of differential equations 548 with respect to time $t$,

$$
\frac{\mathrm{d} S}{\mathrm{~d} t}=-b S I, \quad \frac{\mathrm{d} I}{\mathrm{~d} t}=b S I-g I, \quad \frac{\mathrm{d} R}{\mathrm{~d} t}=g I .
$$

The transition rates of the SIR model define the basic reproduction number of the epidemic, $R_{0}=b / g$. From a phylodynamic standpoint, if the population dynamics of a pathogen is described as a birth-death model with divergence rate $\lambda$ and extinction rate $\mu$, then $R_{t}=\lambda / \mu$ or, alternatively, $R_{t}=\frac{\lambda-\mu}{g}+1^{54}$. We simulated the epidemiological 554 impact of each individual intervention in SIR models with $R_{0}=3$ and $g^{-1}=2$ weeks based on previous estimates ${ }^{24,25}$, yielding a baseline infection rate $b=g R_{0}=6$. In each model, the effective infection rate changed from $b$ to $b \cdot \exp \beta$ on the implementation date of an intervention with log-hazard ratio $\beta$. To determine realistic implementation delays, the starting time of the simulation was set at the date of the first local divergence event in each country and the implementation date was set to the observed median delay across countries (see Fig. 3a). All models started with 100 infected individuals at $t=0$, a value assumed to reflect the number of unobserved cases at the date of the first divergence event, based on the temporality between the divergence events and the reported cases (Extended Data Fig. 3) and on a previous estimate from the U.S. 
564 suggesting that the total number of cases might be two orders of magnitude larger than 565 the reported count ${ }^{55}$. Evaluation of the SIR models used the R package deSolve.

566 Data and software availability

$567 \quad$ All data and software code used to generate the results are available at 568 github.com/rasigadelab/covid-npi. 
570

571

572

573

574

575

576

577

578

579

580

581

582

583

584

585

586

587

588

589

590

591

592

593

594

595

596

597

598

599

600

601

602

603

604

605

606

607

608

1. Wu, F., Zhao, S., Yu, B., Chen, Y.-M., Wang, W., Song, Z.-G., Hu, Y., Tao, Z.-W., Tian, J.-H., Pei, Y.-Y., Yuan, M.-L., Zhang, Y.-L., Dai, F.-H., Liu, Y., Wang, Q.-M., Zheng, J.-J., Xu, L., Holmes, E. C. \& Zhang, Y.-Z. A new coronavirus associated with human respiratory disease in China. Nature 579, 265-269 (2020).

2. Zhou, P., Yang, X.-L., Wang, X.-G., Hu, B., Zhang, L., Zhang, W., Si, H.-R., Zhu, Y., Li, B., Huang, C.-L., Chen, H.-D., Chen, J., Luo, Y., Guo, H., Jiang, R.-D., Liu, M.Q., Chen, Y., Shen, X.-R., Wang, X., Zheng, X.-S., Zhao, K., Chen, Q.-J., Deng, F., Liu, L.-L., Yan, B., Zhan, F.-X., Wang, Y.-Y., Xiao, G.-F. \& Shi, Z.-L. A pneumonia outbreak associated with a new coronavirus of probable bat origin. Nature 579, 270 273 (2020).

3. Zhu, N., Zhang, D., Wang, W., Li, X., Yang, B., Song, J., Zhao, X., Huang, B., Shi, W., Lu, R., Niu, P., Zhan, F., Ma, X., Wang, D., Xu, W., Wu, G., Gao, G. F. \& Tan, W. A Novel Coronavirus from Patients with Pneumonia in China, 2019. New England Journal of Medicine 382, 727-733 (2020).

4. Brauner, J. M., Mindermann, S., Sharma, M., Stephenson, A. B., Gavenčiak, T., Johnston, D., Leech, G., Salvatier, J., Altman, G., Norman, A. J., Monrad, J. T., Besiroglu, T., Ge, H., Mikulik, V., Hartwick, M. A., Teh, Y. W., Chindelevitch, L., Gal, Y. \& Kulveit, J. The effectiveness of eight nonpharmaceutical interventions against COVID-19 in 41 countries. medRxiv 2020.05.28.20116129 (2020). doi:10.1101/2020.05.28.20116129

5. Flaxman, S., Mishra, S., Gandy, A., Unwin, H. J. T., Mellan, T. A., Coupland, H., Whittaker, C., Zhu, H., Berah, T., Eaton, J. W., Monod, M., Ghani, A. C., Donnelly, C. A., Riley, S. M., Vollmer, M. A. C., Ferguson, N. M., Okell, L. C. \& Bhatt, S. Estimating the effects of non-pharmaceutical interventions on COVID-19 in Europe. Nature 1-8 (2020). doi:10.1038/s41586-020-2405-7

6. Li, Y., Campbell, H., Kulkarni, D., Harpur, A., Nundy, M., Wang, X. \& Nair, H. The temporal association of introducing and lifting non-pharmaceutical interventions with the time-varying reproduction number $(R)$ of SARS-CoV-2: a modelling study across 131 countries. The Lancet Infectious Diseases 0, (2020).

7. Bayham, J. \& Fenichel, E. P. Impact of school closures for COVID-19 on the US health-care workforce and net mortality: a modelling study. The Lancet Public Health 0, (2020).

8. Dehning, J., Zierenberg, J., Spitzner, F. P., Wibral, M., Neto, J. P., Wilczek, M. \& Priesemann, V. Inferring change points in the spread of COVID-19 reveals the effectiveness of interventions. Science (2020). doi:10.1126/science.abb9789

9. Flaxman, S., Mishra, S., Gandy, A., Unwin, H., Coupland, H., Mellan, T., Zhu, H., Berah, T., Eaton, J., Perez Guzman, P., Schmit, N., Cilloni, L., Ainslie, K., Baguelin, M., Blake, I., Boonyasiri, A., Boyd, O., Cattarino, L., Ciavarella, C., Cooper, L., Cucunuba Perez, Z., Cuomo-Dannenburg, G., Dighe, A., Djaafara, A., Dorigatti, I., 
609

610

611

612

613

614

615

616

617

618

619

620

621

622

623

624

625

626

627

628

629

630

631

632

633

634

635

636

637

638

639

640

641

642

643

644

645

646

647

Van Elsland, S., Fitzjohn, R., Fu, H., Gaythorpe, K., Geidelberg, L., Grassly, N., Green, W., Hallett, T., Hamlet, A., Hinsley, W., Jeffrey, B., Jorgensen, D., Knock, E., Laydon, D., Nedjati Gilani, G., Nouvellet, P., Parag, K., Siveroni, I., Thompson, H., Verity, R., Volz, E., Walters, C., Wang, H., Wang, Y., Watson, O., Winskill, P., Xi, X., Whittaker, C., Walker, P., Ghani, A., Donnelly, C., Riley, S., Okell, L., Vollmer, M., Ferguson, N. \& Bhatt, S. Report 13: Estimating the number of infections and the impact of non-pharmaceutical interventions on COVID-19 in 11 European countries. (Imperial College London, 2020). doi:10.25561/77731

10. Banholzer, N., Weenen, E. van, Kratzwald, B., Seeliger, A., Tschernutter, D., Bottrighi, P., Cenedese, A., Salles, J. P., Vach, W. \& Feuerriegel, S. Impact of nonpharmaceutical interventions on documented cases of COVID-19. medRxiv 2020.04.16.20062141 (2020). doi:10.1101/2020.04.16.20062141

11. Volz, E. M., Koelle, K. \& Bedford, T. Viral phylodynamics. PLoS Comput. Biol. 9, e1002947 (2013).

12. Paradis, E. Assessing temporal variations in diversification rates from phylogenies: estimation and hypothesis testing. Proceedings of the Royal Society of London. Series B: Biological Sciences 264, 1141-1147 (1997).

13. Rabosky, D. L. Likelihood Methods for Detecting Temporal Shifts in Diversification Rates. Evolution 60, 1152-1164 (2006).

14. The effective reproduction number of an epidemic can be interpreted as the average number of new infections directly caused by a single infected patient. The effective reproduction number equals the basic reproduction number $\mathrm{R} 0$ in a fully susceptible population when no mitigation strategy is active.

15. Tj, N., Tw, K., Jb, K., Cb, D. \& Dp, P. Speciation in North American black basses, Micropterus (Actinopterygii: Centrarchidae). Evolution 57, 1610-1621 (2003).

16. Ma, Z. \& Krings, A. W. Survival Analysis Modeling of Phylogenetic and Coalescent Trees. in 2008 International Conference on BioMedical Engineering and Informatics 1, 178-185 (2008).

17. Nee, S. Inferring Speciation Rates from Phylogenies. Evolution 55, 661-668 (2001).

18. Fisher, L. D. \& Lin, D. Y. Time-dependent covariates in the Cox proportionalhazards regression model. Annu Rev Public Health 20, 145-157 (1999).

19. Stadler, T., Kühnert, D., Bonhoeffer, S. \& Drummond, A. J. Birth-death skyline plot reveals temporal changes of epidemic spread in HIV and hepatitis $\mathrm{C}$ virus (HCV).

Proc. Natl. Acad. Sci. U.S.A. 110, 228-233 (2013).

20. Hadfield, J., Megill, C., Bell, S. M., Huddleston, J., Potter, B., Callender, C., Sagulenko, P., Bedford, T. \& Neher, R. A. Nextstrain: real-time tracking of pathogen evolution. Bioinformatics 34, 4121-4123 (2018).

21. Shu, Y. \& McCauley, J. GISAID: Global initiative on sharing all influenza data - from vision to reality. Euro Surveill. 22, (2017). 
22. Hale, T., Webster, S., Petherick, A., Philips, T. \& Kira, B. Coronavirus Government Response Tracker. Oxford COVID-19 Government Response Tracker, Blavatnik School of Government. Data use policy: Creative Commons Attribution CC BY standard. (2020). at <https://www.bsg.ox.ac.uk/research/researchprojects/coronavirus-government-response-tracker>

23. Ali, S. T., Wang, L., Lau, E. H. Y., Xu, X.-K., Du, Z., Wu, Y., Leung, G. M. \& Cowling, B. J. Serial interval of SARS-CoV-2 was shortened over time by nonpharmaceutical interventions. Science (2020). doi:10.1126/science.abc9004

24. Xu, K., Chen, Y., Yuan, J., Yi, P., Ding, C., Wu, W., Li, Y., Ni, Q., Zou, R., Li, X., Xu, M., Zhang, Y., Zhao, H., Zhang, X., Yu, L., Su, J., Lang, G., Liu, J., Wu, X., Guo, Y., Tao, J., Shi, D., Yu, L., Cao, Q., Ruan, B., Liu, L., Wang, Z., Xu, Y., Liu, Y., Sheng, J. \& Li, L. Factors Associated With Prolonged Viral RNA Shedding in Patients with Coronavirus Disease 2019 (COVID-19). Clin Infect Dis doi:10.1093/cid/ciaa351

25. Bi, Q., Wu, Y., Mei, S., Ye, C., Zou, X., Zhang, Z., Liu, X., Wei, L., Truelove, S. A., Zhang, T., Gao, W., Cheng, C., Tang, X., Wu, X., Wu, Y., Sun, B., Huang, S., Sun, Y., Zhang, J., Ma, T., Lessler, J. \& Feng, T. Epidemiology and transmission of COVID-19 in 391 cases and 1286 of their close contacts in Shenzhen, China: a retrospective cohort study. The Lancet Infectious Diseases 0, (2020).

26. Zhang, J., Litvinova, M., Liang, Y., Wang, Y., Wang, W., Zhao, S., Wu, Q., Merler, S., Viboud, C., Vespignani, A., Ajelli, M. \& Yu, H. Changes in contact patterns shape the dynamics of the COVID-19 outbreak in China. Science 368, 1481-1486 (2020).

27. Salje, H., Tran Kiem, C., Lefrancq, N., Courtejoie, N., Bosetti, P., Paireau, J., Andronico, A., Hozé, N., Richet, J., Dubost, C.-L., Le Strat, Y., Lessler, J., LevyBruhl, D., Fontanet, A., Opatowski, L., Boelle, P.-Y. \& Cauchemez, S. Estimating the burden of SARS-CoV-2 in France. Science (2020). doi:10.1126/science.abc3517

28. Davies, N. G., Kucharski, A. J., Eggo, R. M., Gimma, A., Edmunds, W. J. \& Centre for the Mathematical Modelling of Infectious Diseases COVID-19 working group. Effects of non-pharmaceutical interventions on COVID-19 cases, deaths, and demand for hospital services in the UK: a modelling study. Lancet Public Health 5, e375-e385 (2020).

29. Heavey, L., Casey, G., Kelly, C., Kelly, D. \& McDarby, G. No evidence of secondary transmission of COVID-19 from children attending school in Ireland, 2020. Eurosurveillance 25, 2000903 (2020).

30. Davies, N. G., Klepac, P., Liu, Y., Prem, K., Jit, M. \& Eggo, R. M. Age-dependent effects in the transmission and control of COVID-19 epidemics. Nature Medicine 1-7 (2020). doi:10.1038/s41591-020-0962-9

31. Gonzalez, T., de la Rubia, M. A., Hincz, K. P., Comas-Lopez, M., Subirats, L., Fort, S. \& Sacha, G. M. Influence of COVID-19 confinement on students' performance in higher education. PLoS One 15, e0239490 (2020).

32. Ashikalli, L., Carroll, W. \& Johnson, C. The indirect impact of COVID-19 on child health. Paediatr Child Health (Oxford) (2020). doi:10.1016/j.paed.2020.09.004 
33. Fafi-Kremer, S., Bruel, T., Madec, Y., Grant, R., Tondeur, L., Grzelak, L., Staropoli, I., Anna, F., Souque, P., Mutter, C., Collongues, N., Bolle, A., Velay, A., Lefebvre, N., Mielcarek, M., Meyer, N., Rey, D., Charneau, P., Hoen, B., Seze, J. D., Schwartz, O. \& Fontanet, A. Serologic responses to SARS-CoV-2 infection among hospital staff with mild disease in eastern France. medRxiv 2020.05.19.20101832 (2020). doi:10.1101/2020.05.19.20101832

34. Shim, E., Tariq, A., Choi, W., Lee, Y. \& Chowell, G. Transmission potential and severity of COVID-19 in South Korea. Int. J. Infect. Dis. 93, 339-344 (2020).

35. Aschwanden, C. How Superspreading Events Drive Most COVID-19 Spread. Scientific American at <https://www.scientificamerican.com/article/howsuperspreading-events-drive-most-covid-19-spread1/>

36. Bray, S. R. \& Wang, B. Forecasting unprecedented ecological fluctuations. PLoS Comput. Biol. 16, e1008021 (2020).

702

703

704

705

706

707

708

709

710

711

712

713

714

37. Kain, M. P., Childs, M. L., Becker, A. D. \& Mordecai, E. A. Chopping the tail: how preventing superspreading can help to maintain COVID-19 control. medRxiv 2020.06.30.20143115 (2020). doi:10.1101/2020.06.30.20143115

38. Rapid Risk Assessment: Coronavirus disease 2019 (COVID-19) in the EU/EEA and the UK - tenth update. European Centre for Disease Prevention and Control (2020). at <https://www.ecdc.europa.eu/en/publications-data/rapid-risk-assessmentcoronavirus-disease-2019-covid-19-pandemic-tenth-update>

39. Lemey, P., Hong, S. L., Hill, V., Baele, G., Poletto, C., Colizza, V., O'Toole, Á., McCrone, J. T., Andersen, K. G., Worobey, M., Nelson, M. I., Rambaut, A. \& Suchard, M. A. Accommodating individual travel history and unsampled diversity in Bayesian phylogeographic inference of SARS-CoV-2. Nat Commun 11, 5110 (2020).

40. Variation in government responses to COVID-19. at $<$ https://www.bsg.ox.ac.uk/research/publications/variation-government-responsescovid-19>

41. Paradis, E. Statistical Analysis of Diversification with Species Traits. Evolution 59, 1-12 (2005).

42. Biek, R., Pybus, O. G., Lloyd-Smith, J. O. \& Didelot, X. Measurably evolving pathogens in the genomic era. Trends Ecol. Evol. (Amst.) 30, 306-313 (2015).

43. nextstrain/ncov. (Nextstrain, 2020). at <https://github.com/nextstrain/ncov>

44. Minh, B. Q., Schmidt, H. A., Chernomor, O., Schrempf, D., Woodhams, M. D., von Haeseler, A. \& Lanfear, R. IQ-TREE 2: New Models and Efficient Methods for Phylogenetic Inference in the Genomic Era. Mol. Biol. Evol. 37, 1530-1534 (2020).

45. Sagulenko, P., Puller, V. \& Neher, R. A. TreeTime: Maximum-likelihood phylodynamic analysis. Virus Evol 4, (2018).

46. Orientation: so, what does Nextstrain do? Tutorial: Using Nextstrain for SARS-CoV2 at <https://nextstrain.github.io/ncov/orientation-workflow.html> 
47. Therneau, T. M. \& Grambsch, P. M. Modeling Survival Data: Extending the Cox Model. (Springer Science \& Business Media, 2013).

48. Pagel, M. The Maximum Likelihood Approach to Reconstructing Ancestral Character States of Discrete Characters on Phylogenies. Syst Biol 48, 612-622 (1999).

49. Austin, P. C. A Tutorial on Multilevel Survival Analysis: Methods, Models and Applications. International Statistical Review 85, 185-203 (2017).

50. Nee, S., May, R. M. \& Harvey, P. H. The reconstructed evolutionary process. Philos. Trans. R. Soc. Lond., B, Biol. Sci. 344, 305-311 (1994).

51. Hedges, S. B., Marin, J., Suleski, M., Paymer, M. \& Kumar, S. Tree of Life Reveals Clock-Like Speciation and Diversification. Mol Biol Evol 32, 835-845 (2015).

52. Etienne, R. S. \& Rosindell, J. Prolonging the past counteracts the pull of the present: protracted speciation can explain observed slowdowns in diversification. Syst. Biol. 61, 204-213 (2012).

53. Nabhan, A. R. \& Sarkar, I. N. The impact of taxon sampling on phylogenetic inference: a review of two decades of controversy. Brief Bioinform 13, 122-134 (2012).

54. Pybus, O. G., Charleston, M. A., Gupta, S., Rambaut, A., Holmes, E. C. \& Harvey, P. H. The epidemic behavior of the hepatitis $C$ virus. Science 292, 2323-2325 (2001).

55. Silverman, J. D., Hupert, N. \& Washburne, A. D. Using influenza surveillance networks to estimate state-specific prevalence of SARS-CoV-2 in the United States. Science Translational Medicine (2020). doi:10.1126/scitranslmed.abc1126 
752 Acknowledgements: We thank Philip Supply, François Vandenesch, Jean-Sébastien 753 Casalegno, Vanessa Escuret, and Christophe Ramière for fruitful discussions and 754 reviews of our work. We thank the GISAID, Nextstrain and OxCGRT teams for making 755 their high-quality datasets available to the community. A list of authors and laboratories 756 contributing SARS-CoV-2 genome sequences is shown in Extended Data Table 3.

757 Funding: JPR received support from the FINOVI Foundation (grant R18037CC).

758 Author contributions: JPR, LJ, TW designed research. JPR, ABarray, JTS, CQ, YV, 759 GD, LJ conducted research. JPR, TW analyzed the data. JPR created figures. JPR, Abal, $760 \mathrm{GD}, \mathrm{LJ}, \mathrm{PV}, \mathrm{BL}, \mathrm{TW}$ interpreted the data. All authors wrote the paper.

761 Competing interests: $\mathrm{BL}$ is currently active in groups advising the French government 762 for which $\mathrm{BL}$ is not receiving payment.

763 Data and material availability: Data and analysis code are available online at 764 https://github.com/rasigadelab/covid-npi. 


\section{$765 \quad$ EXTENDED DATA}

766 Extended Data Figures 1 to 8 included below

$767 \quad$ Extended Data Tables 1 and 2 included below

768 Other Supplementary Information for this manuscript include the following:

769 Extended Data Table 3. Number of samples, phylogenetic branches, and dates of first 770 detected SARS-CoV-2 local transmission events and non-pharmaceutical interventions 771 in 74 countries. (.xIsx spreadsheet)

772 Extended Data Table 4. Detailed timeline of implementation and release of non773 pharmaceutical interventions against COVID-19 in 74 countries up to May 12, 2020.

$774 \quad$ (.xlsx spreadsheet)

775 Extended Data Table 5. Authors and laboratories having contributed SARS-CoV-2

776 genomes included in the dated phylogeny. (.xlsx spreadsheet)

777

778

779

780 


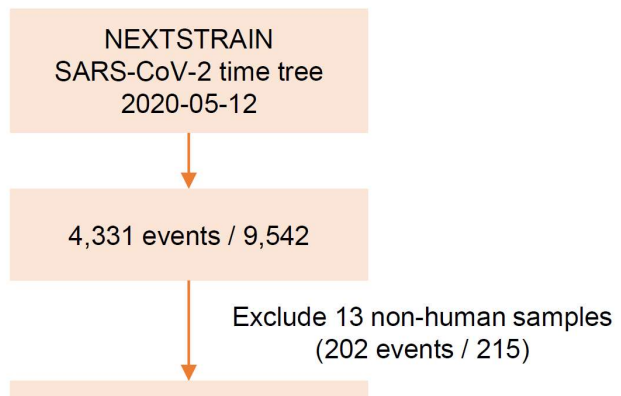

4,129 events / 9,327

Resolve polytomies

5,196 events / 10,394

Exclude uncertain country assignations $(1,171$ events / 2,162$)$

4,025 events / 8,232

included in country comparison model

Exclude events in countries with unknown NPIs (6 events / 22)
27 countries with $\geq 10$ events; 32 countries with $<10$ events grouped in 'Others' category
Oxford COVID-19

Government Response Tracker 2020-05-12

74 countries contributing $\geq 1$ human sample

Exclude 15 countries with no event assigned

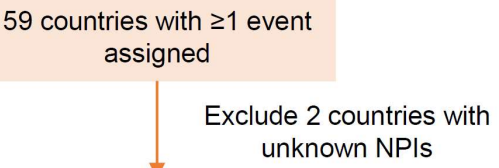

59 countries with $\geq 1$ event assigned

Exclude 2 countries with unknown NPIs

57 countries with known NPIs

Extended Data Figure 1. Flowchart of data selection. Events are phylogenetic divergences (tree nodes in the SARS-CoV-2 phylogeny), excluding tree root. Polytomies are unresolved tree nodes representing $>1$ divergence event. Polytomies were resolved into dichotomies (nodes with exactly 1 divergence) with arbitrarily small interval length. NPI, non-pharmaceutical 


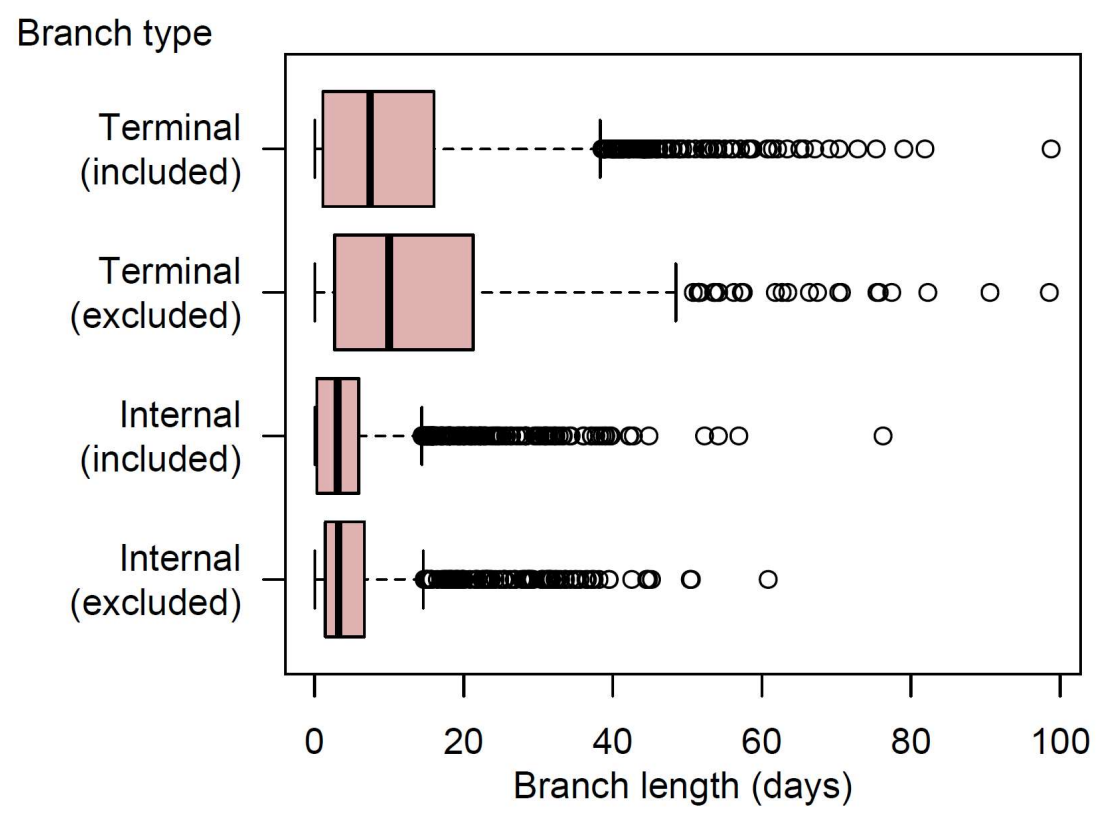

791

792 Extended Data Figure 2. Length distribution in phylogenetic branches with uncertain country assignation. Shown are box-and-whisker plots of the lengths of internal and terminal branches, depending on branch exclusion due to uncertain ( $<95 \%$ confidence) country assignation. Boxes denote interquartile range (IQR) and median, whiskers extend to lengths at most 1.5x the IQR away from the median length, and circle marks denote lengths farther than $1.5 \mathrm{IQR}$ from the median length. 


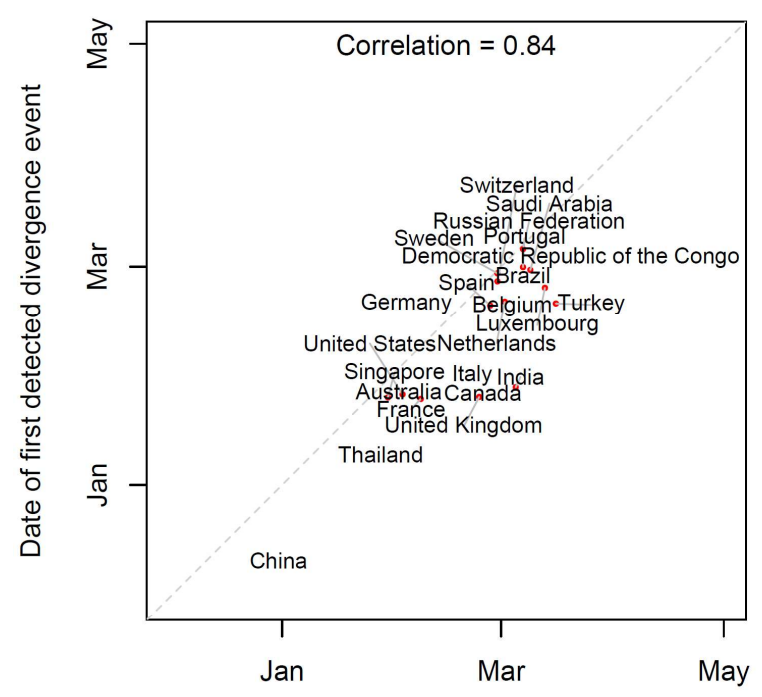

798 799

800 801 802 803 804 with at least 15 assigned internal branches.

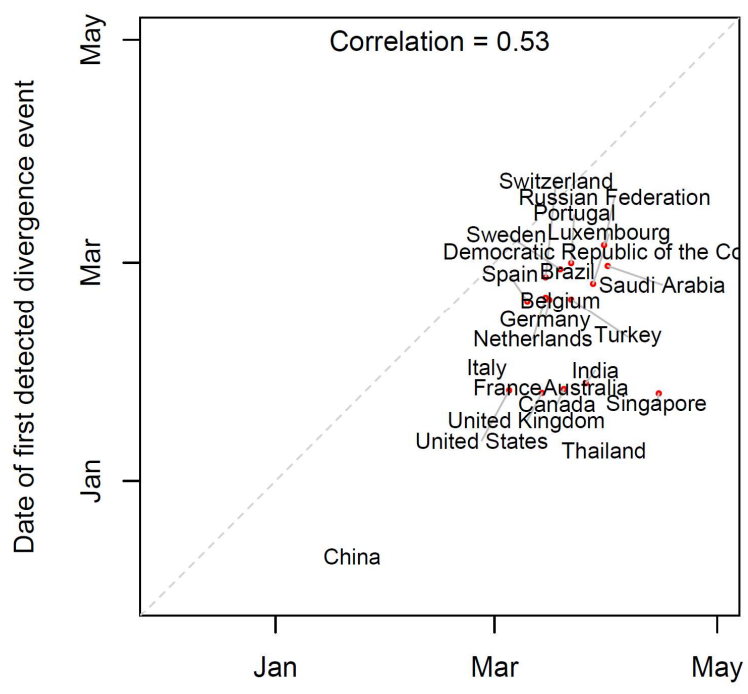

Date of 10 th reported Covid-19 death

Extended Data Figure 3. Correlation of reported and estimated epidemic onset dates. Dates of first estimated autochtonous SARS-CoV-2 transmission per country relative to the dates of the $10^{\text {th }}$ reported case (left panel) and the $10^{\text {th }}$ reported death (right panel) in countries 


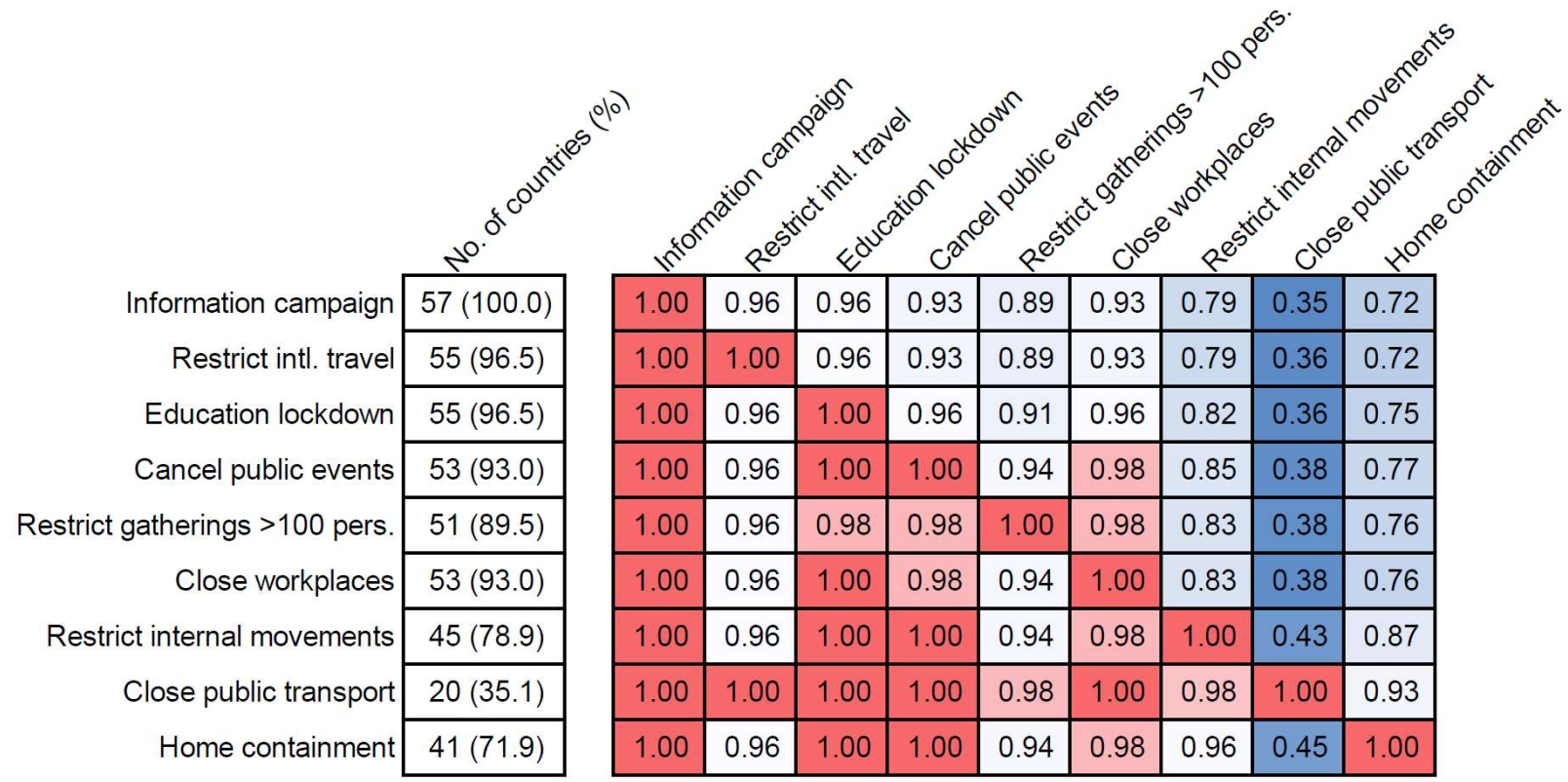
interventions in $\mathbf{5 7}$ countries. The first column shows the number and percentage of countries implementing each intervention, independent of other interventions. Matrix cells show the proportion of countries implementing the intervention in column conditional on the 

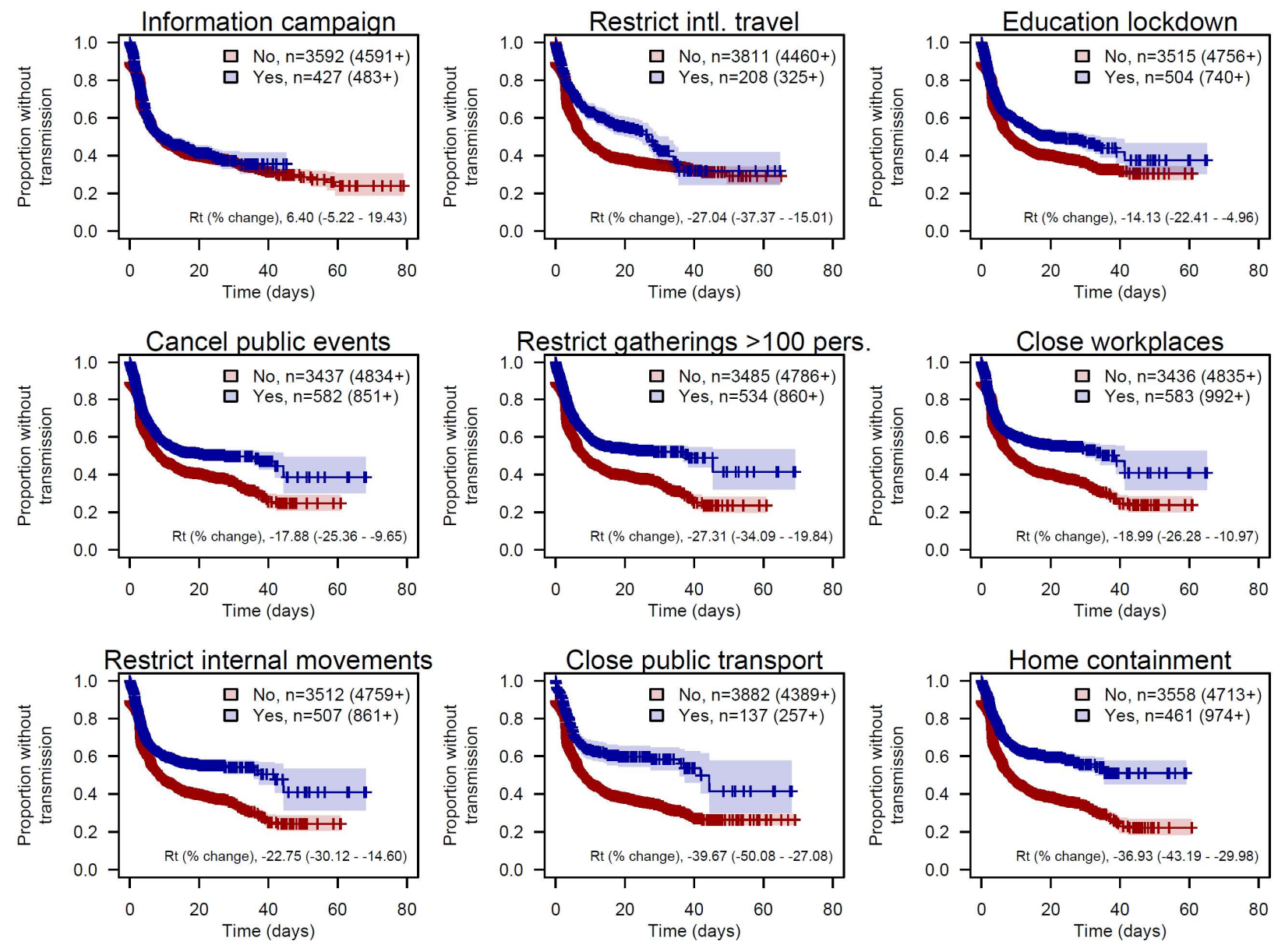
with reduced effective reproduction numbers. Data derive from a dated phylogeny of SARSCoV-2 genomes from 57 countries, with 4,191 internal branches interpreted as time-to-event intervals, and 4,019 terminal branches interpreted as censored intervals, after exclusion of branches with uncertain country assignation. Shown are Kaplan-Meier survival curves of the waiting time without a viral transmission event, stratified on the presence of nine nonpharmaceutical interventions active or not in each country. Sample sizes denote, for each stratum, the no. of time-to-event subintervals (possibly resulting from splitting intervals containing a change of intervention) and, in brackets, the no. of censored subintervals. Percent changes of the effective reproduction number $R_{t}$ were derived from separate time-dependent mixed-effect Cox regression models treating the country and the branch as random effects. 


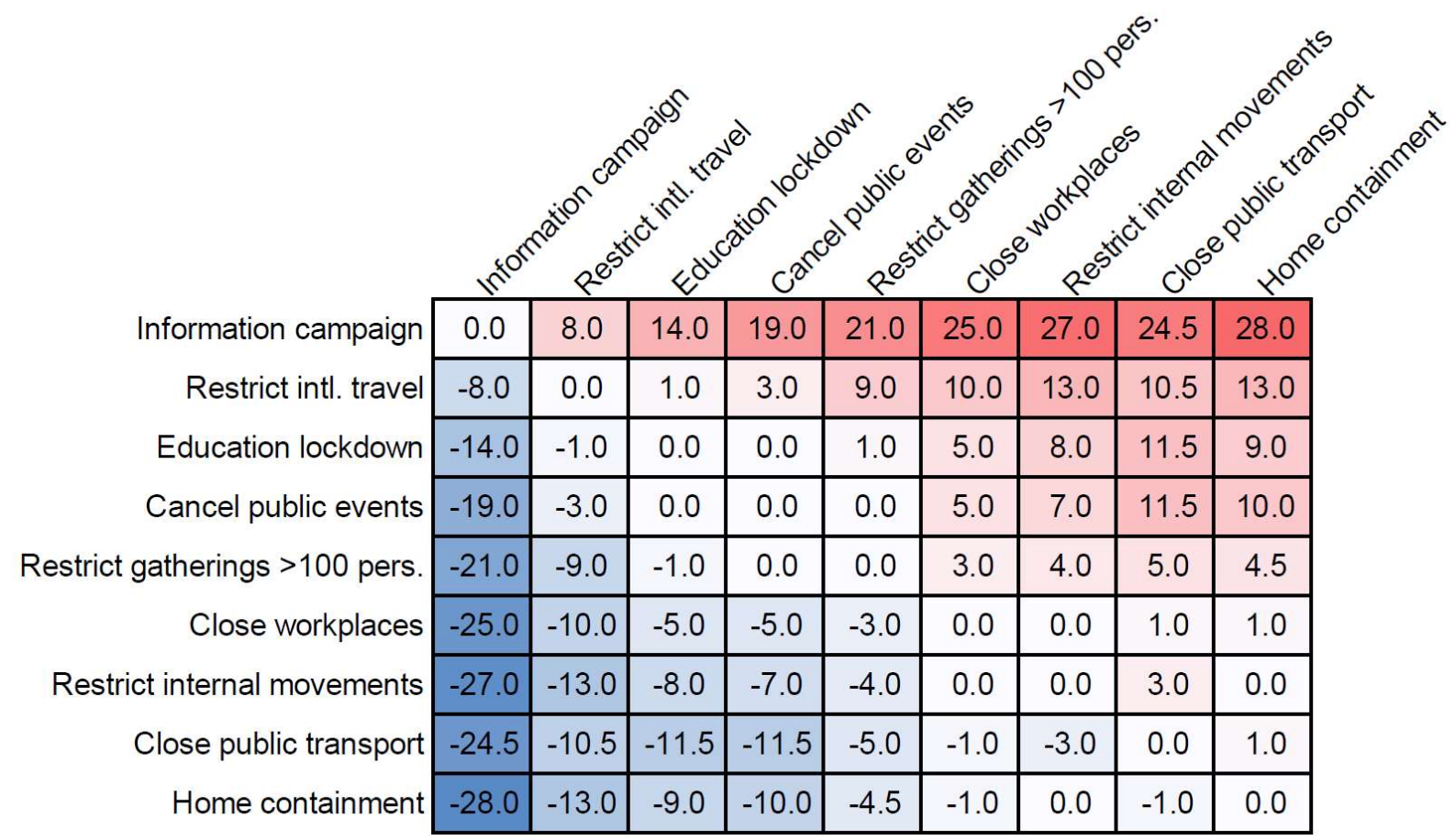
interventions. Shown are the median days elapsed between the implementation of the intervention in the row and that of the intervention in the column, where median is taken across countries that implemented both interventions. 


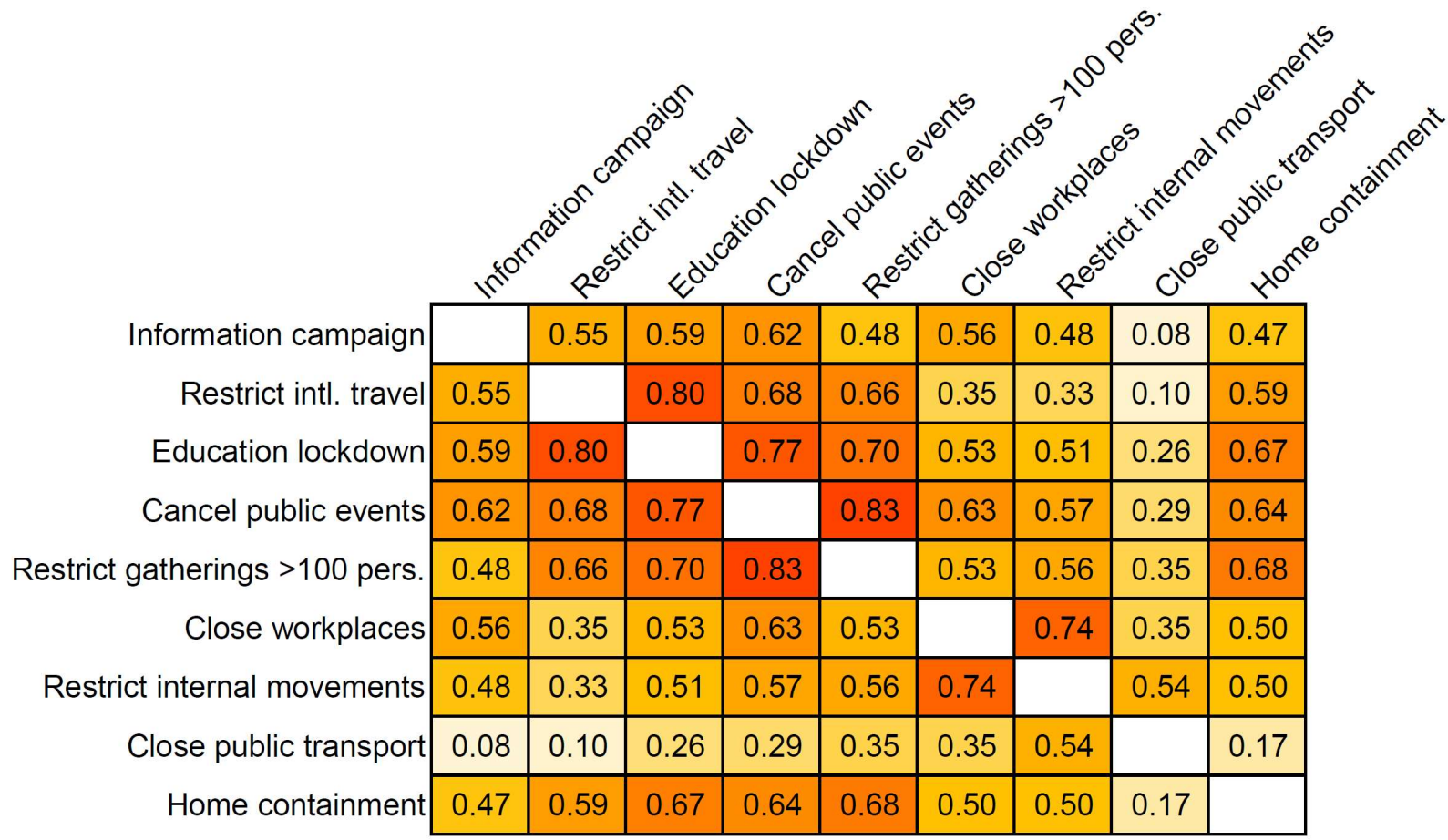
censored sub-intervals. Sub-intervals result from splitting phylogenetic branches $(n=8,210)$ in which a change of intervention (activation or release) occurs. Smaller absolute correlations (white) favor the identifiability of intervention effects in multivariable analysis while larger absolute correlations (orange/red) can result into dependencies between model coefficients (see Fig. 3c). 


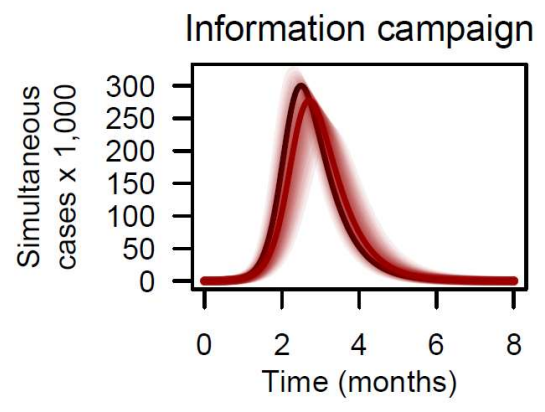

Cancel public events

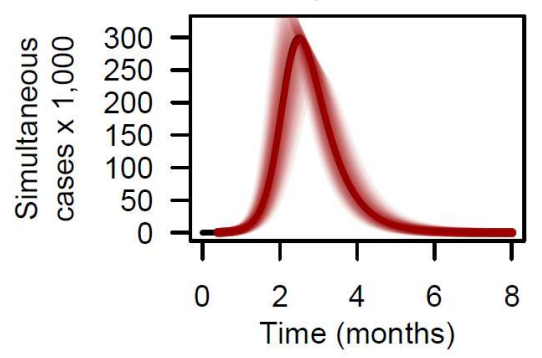

Restrict internal movements

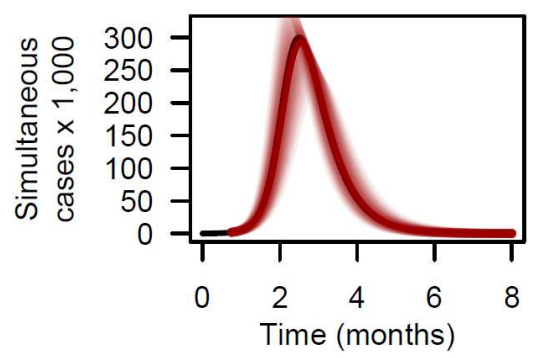

Restrict intl. travel

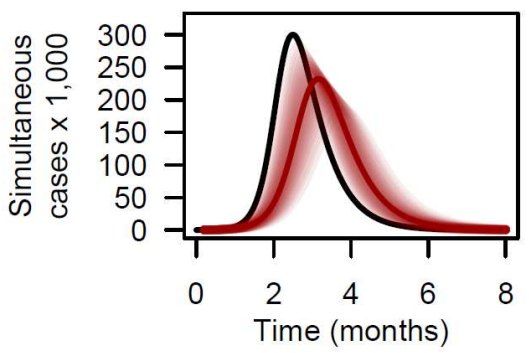

Restrict gatherings

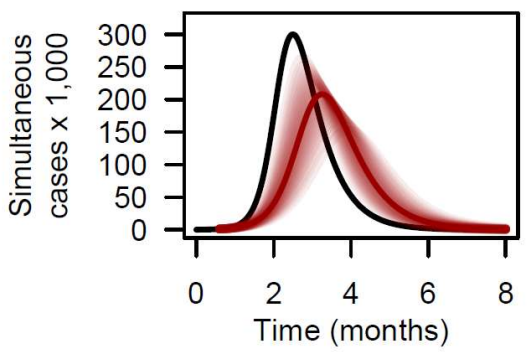

Close public transport

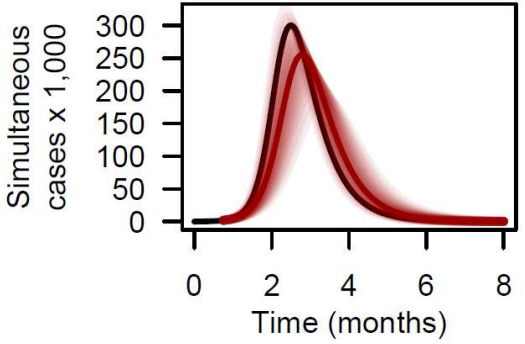

Education lockdown

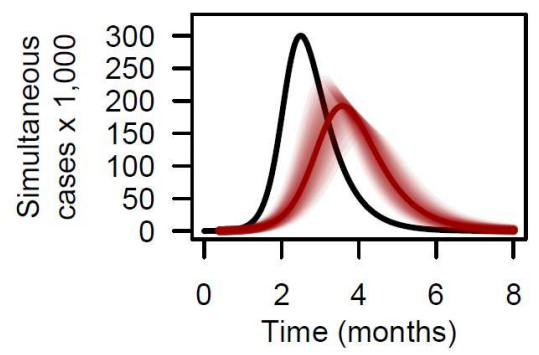

Close workplaces

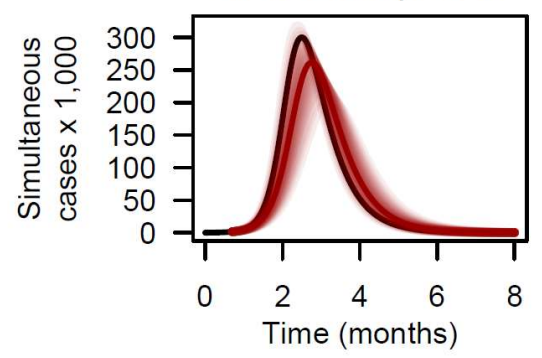

Home containment

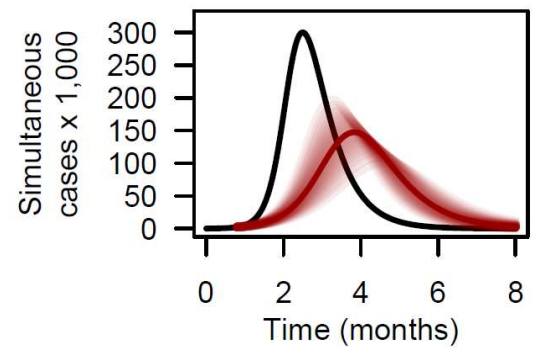

Extended Data Figure 8. Predicted individual impact of 9 non-pharmaceutical interventions (NPIs) on the number of simultaneous COVID-19 cases in an idealized population of 1 million susceptible individuals. Gray lines represent the case count predicted by an epidemiological SIR model with a basic reproduction number $R_{0}=3$, as estimated in the absence of NPIs, and a mean infectious period of 2 weeks. For each NPI, the simultaneous case count (red line) and 95\% confidence band are derived from an SIR model in which the basic reproduction number is altered as predicted by the mutivariate model coefficients shown in Fig. 3b. The delay between the $100^{\text {th }}$ case and NPI implementation in SIR models coincides with the median delay between the $1^{\text {st }}$ transmission event and the NPI implementation shown in Fig. 3a. 
Extended Data Table 1. Predicted percent change of COVID-19 effective reproduction number in response to non-pharmaceutical interventions with and without adjustment for time. Data derive from multivariable mixed-effect Cox regression models including one random intercept per country and phylogenetic branch.

\begin{tabular}{lcc}
\hline $\begin{array}{l}\text { Factor } \\
\text { Elapsed time (per month) }\end{array}$ & \multicolumn{2}{c}{ Relative $R_{t}$ (\% change $)$} \\
$\begin{array}{l}\text { Information campaign } \\
\text { Restrict intl. travel }\end{array}$ & $-6.0(-17.0$ to 6.5$)$ & \multicolumn{1}{c}{ Time-adjusted model } \\
\hline $\begin{array}{l}\text { Education lockdown } \\
\text { Cancel public events }\end{array}$ & $-16.9(-27.5$ to -4.8$)$ & $-11.0(-22.8$ to 2.7$)$ \\
Restrict gatherings $>100$ pers. & $-25.6(-33.4$ to -16.9$)$ & $-21.0(-29.6$ to -11.2$)$ \\
Close workplaces & $-1.0(-14.7$ to 15.0$)$ & $1.1(-13.5$ to 18.0$)$ \\
Restrict internal movements & $-10.0(-22.8$ to 5.0$)$ & $-8.8(-22.2$ to 6.9$)$ \\
Close public transport & $-2.2(-16.8$ to 15.0$)$ & $0.8(-14.8$ to 19.2$)$ \\
Home containment & $-11.5(-26.6$ to 6.7$)$ & $-9.7(-25.6$ to 9.5$)$ \\
\hline
\end{tabular}


Extended Data Table 2. Predicted reduction of the COVID19 effective reproduction number by non-pharmaceutical interventions implemented alone.

\begin{tabular}{lc}
\hline Intervention & $\begin{array}{c}\text { Probability that } \\
\boldsymbol{R}_{\boldsymbol{t}}<\mathbf{1} \text { if } \boldsymbol{R}_{\mathbf{0}}=\mathbf{1 . 5}\end{array}$ \\
\hline Information campaign & $<0.01$ \\
Restrict intl. travel & $<0.01$ \\
Education lockdown & 0.03 \\
Cancel public events & $<0.01$ \\
Restrict gatherings $>100$ pers. & 0.03 \\
Close workplaces & $<0.01$ \\
Restrict internal movements & $<0.01$ \\
Close public transport & $<0.01$ \\
Home containment & 0.61 \\
\hline
\end{tabular}

NOTE. The probability that $R_{t}<1$ was less than 0.01 for all interventions if $R_{0} \geq 2$. 
Figures

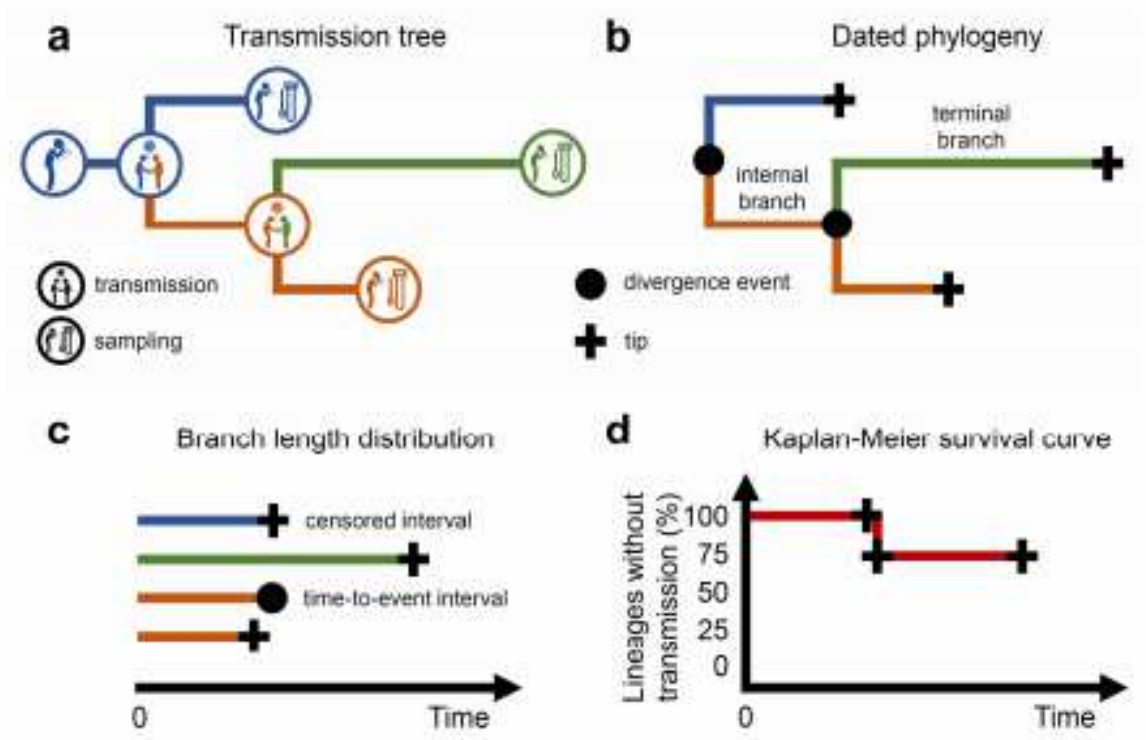

\section{Figure 1}

[Please see the manuscript file to view the figure caption.] 
a

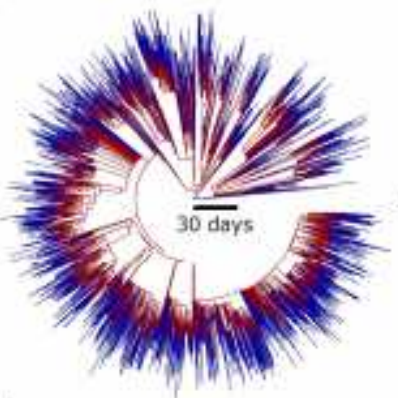

b

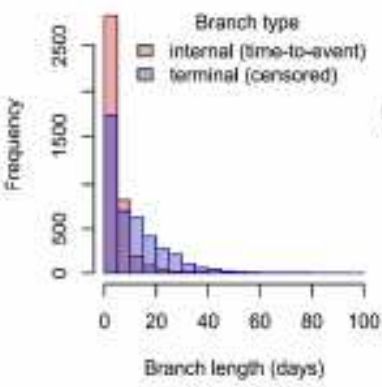

e

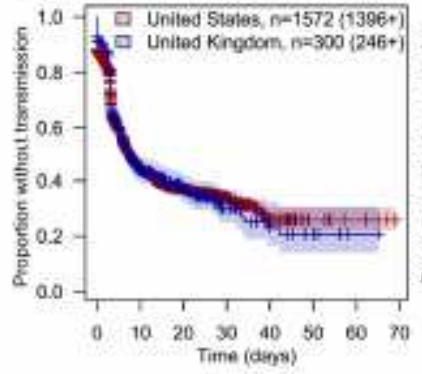

C

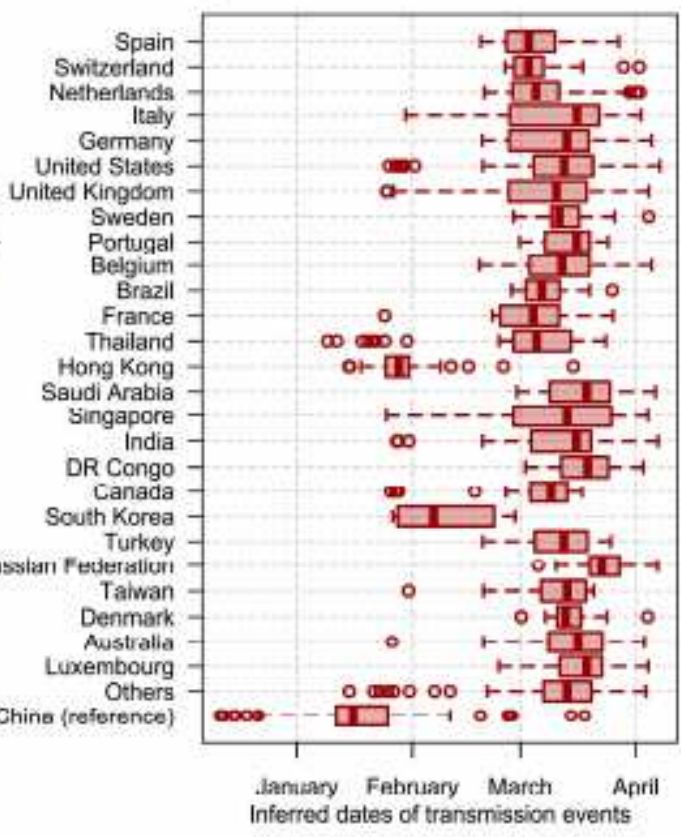

f

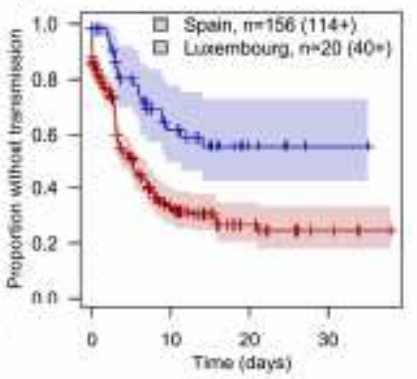

d

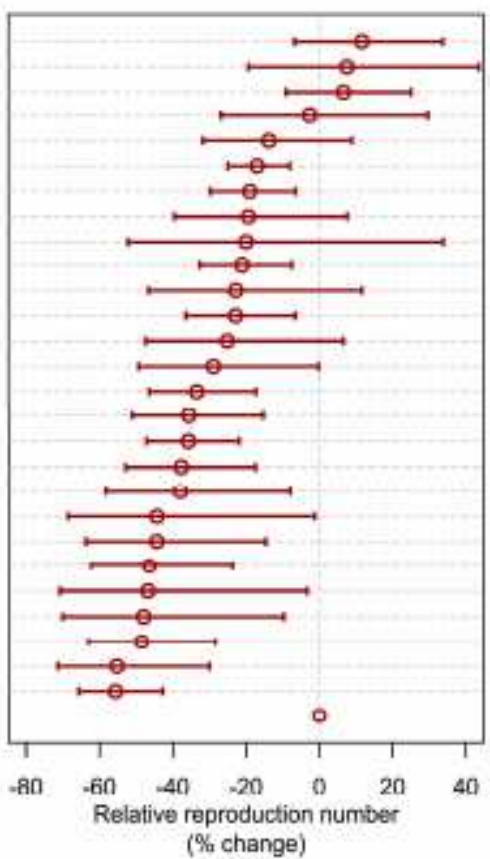

h g

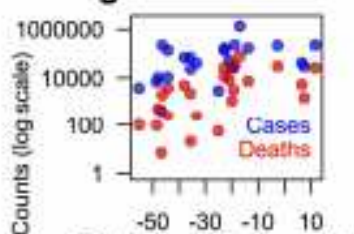

Relative reproduction number (Th charges relative to China)

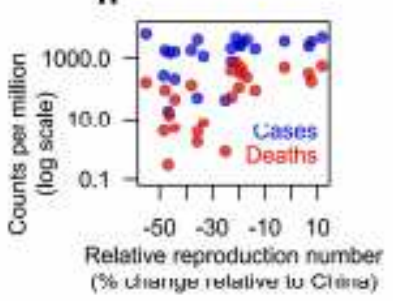

Figure 2

[Please see the manuscript file to view the figure caption.] 
a

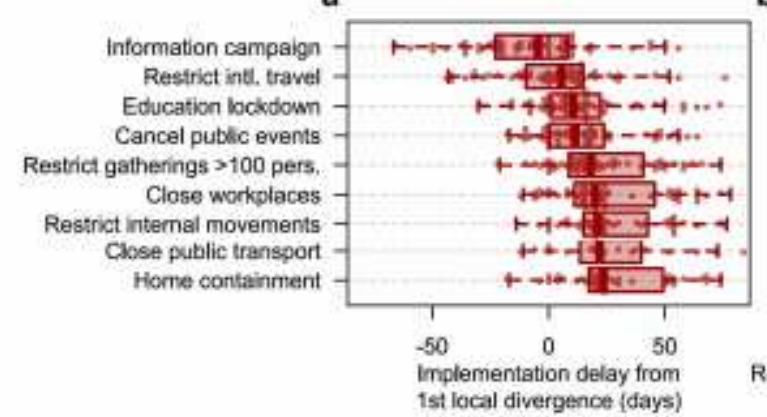

C $\%$ change in full moder b

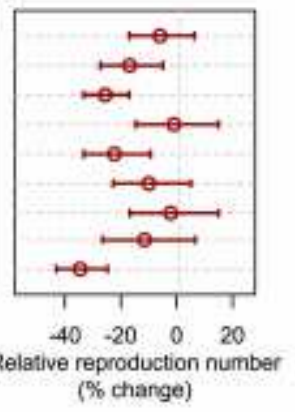

d Home containment Education lockdown
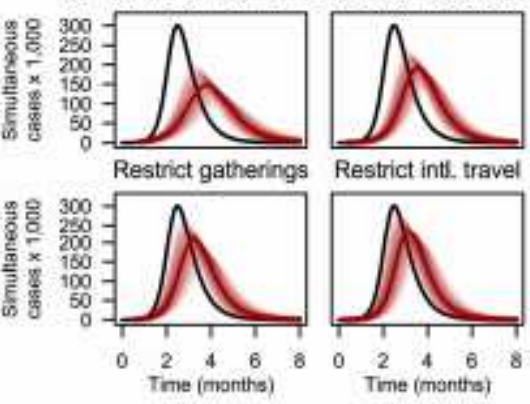

e Cumulative effect of combined interventions

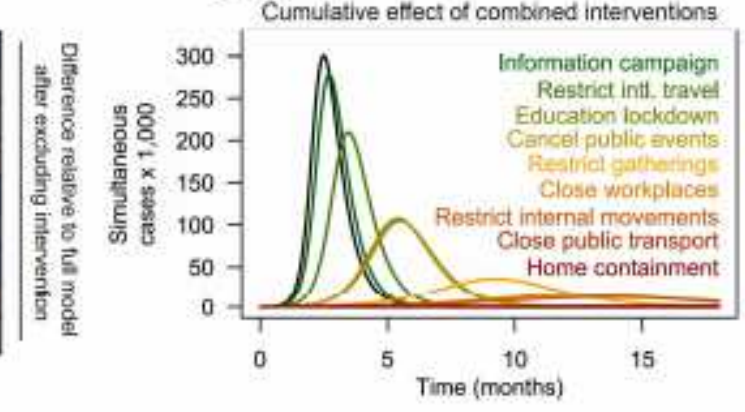

\section{Figure 3}

[Please see the manuscript file to view the figure caption.]

\section{Supplementary Files}

This is a list of supplementary files associated with this preprint. Click to download.

- ExtendedDataTable3.xlsx

- ExtendedDataTable4.xIsx

- ExtendedDataTable5.xlsx 